\title{
Review
}

Tianqi Niu, Qifan Xue* and Hin-Lap Yip*

\section{Advances in Dion-Jacobson phase two-dimensional metal halide perovskite solar cells}

https://doi.org/10.1515/nanoph-2021-0052

Received February 5, 2021; accepted March 17, 2021;

published online April 1, 2021

\begin{abstract}
Low-dimensional metal halide perovskites have emerged as promising alternatives to the traditional threedimensional (3D) components, due to their greater structural tunability and environmental stability. DionJacobson (DJ) phase two-dimensional (2D) perovskites, which are formed by incorporating bulky organic diammonium cations into inorganic frameworks that comprises a symmetrically layered array, have recently attracted increasing research interest. The structureproperty characteristics of DJ phase perovskites endow them with a unique combination of photovoltaic efficiency and stability, which has led to their impressive employment in perovskite solar cells (PSCs). Here, we review the achievements that have been made to date in the exploitation of DJ phase perovskites in photovoltaic applications. We summarize the various ligand designs, optimization strategies and applications of DJ phase PSCs, and examine
\end{abstract}

\footnotetext{
*Corresponding authors: Qifan Xue, State Key Laboratory of Luminescent Materials and Devices, Institute of Polymer Optoelectronic Materials and Devices, School of Materials Science and Engineering, South China University of Technology, Guangzhou 510640, P. R. China; and Innovation Center of Printed Photovoltaics, South China Institute of Collaborative Innovation, Dongguan 523808, P. R. China, E-mail: qfxue@scut.edu.cn; and Hin-Lap Yip, State Key Laboratory of Luminescent Materials and Devices, Institute of Polymer Optoelectronic Materials and Devices, School of Materials Science and Engineering, South China University of Technology, Guangzhou 510640, P. R. China; Innovation Center of Printed Photovoltaics, South China Institute of Collaborative Innovation, Dongguan 523808, P. R. China; Department of Materials Science and Engineering, City University of Hong Kong, Tat Chee Avenue, Kowloon, Hong Kong; and School of Energy and Environment, City University of Hong Kong, Tat Chee Avenue, Kowloon, Hong Kong, E-mail: msangusyip@scut.edu.cn Tianqi Niu, State Key Laboratory of Luminescent Materials and Devices, Institute of Polymer Optoelectronic Materials and Devices, School of Materials Science and Engineering, South China University of Technology, Guangzhou 510640, P. R. China
}

the current understanding of the mechanisms underlying their functional behavior. Finally, we discuss the remaining bottlenecks and future outlook for these promising materials, and possible development directions of further commercial processes.

Keywords: advances; Dion-Jacobson phase; solar cells; two-dimensional perovskites.

\section{Introduction}

Metal halide perovskites have attracted tremendous attention due to their excellent optoelectronic properties, which include long carrier-diffusion lengths, high absorption coefficients, high defect tolerances, tunable bandgaps and a capacity for ambipolar charge transport [1-5]. In addition, they can be fabricated via facile lowtemperature deposition processes, which benefits to the inexpensive and scalable preparation. Miyasaka et al. first reported the utility of metal halide perovskites in photovoltaic applications in 2009, with a device that exhibited an efficiency of 3.8\% [6], and in the subsequent decade the certified efficiency of perovskite solar cells (PSCs) has rapidly increased to as high as $25.5 \%$ [7]. The excellent optoelectronic properties of perovskites have seen them become among the best-performing third-generation thinfilm materials for employment in photovoltaic fields. However, the practical application of perovskites remains limited by the inherently poor stability of their hybrid organic-inorganic frameworks, especially under longterm moisture, heat and illumination stresses [8]. This poor environmental resistance primarily results from their susceptibility to light-induced ionic diffusion and the volatility of the organic cations. Moreover, exposure to moisture triggers irreversible transitions of photo-active perovskite phases, which inhibits the improvement of corresponding device performance [9]. Additional perovskite degradation pathways are activated by exposure to electric fields, molecular oxygen, UV irradiation and other conditions, which demonstrates the environmental weakness of these materials [10-12]. 
In contrast to the volatile small cations such as methylammonium $\left(\mathrm{MA}^{+}\right)$and formamidinium $\left(\mathrm{FA}^{+}\right)$that are used in traditional three-dimensional (3D) perovskite components, the bulky organic cations incorporated within two-dimensional (2D) perovskites exhibit greater environmental resistance [13, 14]. The incorporation of certain long-chain organic cations into perovskite lattice could slice the inorganic sheet layer to adopt a lowdimensional layered configuration, thereby distorting the structural integrity of the inorganic octahedron framework [15]. The 2D perovskites have a multiple quantum-well $(\mathrm{QW})$ structure and the general formula $\left(\mathrm{A}^{\prime}\right)_{m}(\mathrm{~A})_{n-1} \mathrm{M}_{n} \mathrm{X}_{3 n+1}$, where $\mathrm{A}^{\prime}$ is a bulky mono- or divalent spacer cation between the perovskite layers, $\mathrm{M}$ is a divalent metal ion, which is commonly a lead or tin cation $\left(\mathrm{Pb}^{2+}\right.$ or $\left.\mathrm{Sn}^{2+}\right)$, and $\mathrm{X}$ represents a halide anion $[16,17]$. The $\mathrm{A}^{+}, \mathrm{M}^{2+}$ and $\mathrm{X}^{-}$ components form the octahedron framework and $n$ refers to the thickness of the $\left[\mathrm{MX}_{6}\right]^{4-}$ inorganic layers, which are connected via van der Waals interactions or by hydrogen bonds between adjacent spacer cations. The QW width is determined by the value of $n$, which can be controlled by stoichiometric manipulation, whereas the interlayer distance depends on the length of the spacer cations and spatial effects. The presence of bulky hydrophobic organic cations in 2D perovskites has been shown to strain the surface-level $\mathrm{Pb}$-X bonds and hinder water adsorption onto reactive sites [18]. In addition, dimensional tailoring was found to gradually lift the size restraints imposed by the Goldschmidt tolerance factor, leading to the unique structural tunability of 2D perovskites [19, 20]. More and more organic spacer cations have been chosen to synthesize and modify the new-type 2D perovskites [21]. The structural variation induced by ligand chemistry shows a significant influence on QW distribution, electronic structure and optoelectronic properties of 2D perovskites. Among them, the Dion-Jacobson (DJ) phase 2D perovskites containing the single diammonium spacer cation as interlayer absolutely eliminate the weak van der Waals interaction between alternating inorganic layers [22]. The strong hydrogenbonding interaction between the spacer layers and adjacent inorganic layers in $\mathrm{DJ}$ phase $2 \mathrm{D}$ perovskites give them a more stable framework and a shorter interlayer distance than their Ruddlesden-Popper (RP) phase analogs, and reduces the barrier to charge transport in their organic layers.

Layered perovskites (a collective reference here for the 2D and quasi-2D perovskites) have been extensively utilized in photovoltaic solar cells, and the first 2D (PEA) $(\mathrm{MA})_{2} \mathrm{~Pb}_{3} \mathrm{I}_{10}$-based metal halide PSC was formed by incorporating the bulky phenylethylammonium (PEA) cation into 3D $\mathrm{MAPbI}_{3}$. The device exhibited a relatively low power conversion efficiency (PCE) of $4.7 \%$, but had much better ambient stability than its 3D analogue [13]. Subsequently, the hot-casting method was a key breakthrough in the development of high-performance 2D PSCs, as it enabled near-single-crystalline film quality and preferentially vertical crystal orientation to be achieved, resulting in a device PCE of 12.51\% [23]. Extensive research has since been conducted to further improve the performance of $2 \mathrm{D}$ PSCs, in areas such as procedural optimization [23, 24], solvent engineering $[25,26]$, composition engineering [14, $27,28]$ and ligand tailoring [21]. The current world-record PCE of RP phase PSCs $(n \leq 5)$ has achieved over 18\% [29-32]. Recently, Zhang et al. used amylamine (AA) as spacer cation and further refreshed the record up to be $18.42 \%$ based on (AA) ${ }_{2} \mathrm{MA}_{3} \mathrm{~Pb}_{4} \mathrm{I}_{13}$ cells [33]. By contrast, the development time of DJ phase PSCs is shorter than those relatively mature RP phase systems. In 2018, Kanatzidis et al. first reported DJ phase 2D perovskite structures $(n=1-$ 4) based on 3AMP and 4AMP cations [AMP = (aminomethyl)piperidinium], and the corresponding PSCs achieved a PCE of 7.32\% [22]. Since then, the PCEs of DJ phase PSCs have increased substantially to as high as $18.2 \%$, which is competitive with their RP phase counterparts [34]. Besides, the stability advantages of DJ phase 2D PSCs under varied harsh environmental conditions, etc. humidity, heat and illumination, are also highlighted due to their good structural stability as the stronger electrostatic interaction between adjacent interlayers than RP phase analogs $[35,36]$. Hence, DJ phase perovskites are promising light-harvesting materials for application in highly stable solar cells comprising a broad range of chemical compositions [37].

However, the main challenge of DJ phase or whole 2D perovskites is the current efficiency bottleneck, which lags far behind those of their 3D analogs. This suboptimal optoelectronic behavior of $\mathrm{DJ}$ phase $2 \mathrm{D}$ perovskites is due to a common problem in the development of low-dimensional perovskites: the increased quantum and dielectric confinement that occurs when crystal dimensions are decreased. This results in DJ phase 2D perovskites occupying with narrower absorption windows and higher exciton-binding energies than 3D perovskites. Moreover, films formed from solution-processed DJ phase 2D perovskites suffer from random crystal orientations and broad phase distribution, which decreases oriented charge transport and induces severe charge accumulation and recombination loss. Therefore, there is a pressing need for a summary of the current design and optimization strategies for DJ phase 2D PSCs, as this, combined with knowledge gained from the exploration of RP phase perovskites, will spur the development of feasible approaches to improved DJ phase PSCs. In particular, the determination of fundamental structure-property relationships and 
advancements in the preparation of key materials will facilitate self-assembly processes and the regulation of crystallization kinetics, and enable precise control of crystal orientations and phase compositions. This will motivate the development of high-performance DJ phase PSCs that exhibit good efficiency, reproducibility, stability.

In this review, we systematically describe current advances in the employment of $\mathrm{DJ}$ phase 2D perovskites in photovoltaic applications. First, we briefly introduce the chemical and crystal structures of this new category of 2D perovskites, and their optoelectronic properties. We then highlight different ligand designs, optimization strategies and advanced applications, and the mechanisms underlying these, that represent progress toward efficient and stable DJ phase PSCs. Finally, the remaining challenges and future outlook for these promising photovoltaic materials are discussed, to explore effective development directions for further commercial process.

\section{Basic structural and optoelectronic properties}

The sizes and shapes of organic spacer cations in 2D perovskites can be tuned to optimize their QW structures and crystal orientation to achieve desired crystallinities and optoelectronic properties. The abundance of spacer cations provides access to a wide range of layered perovskites, which deepens our understanding of the relationships between perovskite structures and film properties. In addition, the functionalization of organic ligands can lead to the discovery of novel structures.

According to the changed crystallographic directions, the 2D perovskites can be categorized to (100)-, (110)-, and (111)-oriented perovskite structure, defined as the slicing crystallographic planes of the infinitely extending inorganic sheets of the original 3D perovskite structure. The formation of different categories of 2D perovskites depends on the structure and size of the incorporated long-chain organic cations, and leads to a variety of layer-stacking modes. The different categories present the distinct difference in relative stacking mode of layers. Among these three classifications, the (100)-oriented 2D perovskites represent the widely investigated systems for photovoltaic applications, which can be further divided into RP phase, DJ phase and alternating cations in the interlayer space (ACI) phase subcategories (Figure 1A) [39-41]. Aurivillius (AV) perovskites are also belonging to the (100)-oriented 2D perovskites, but appear only in oxide perovskite systems [42, 43]. The (110)- and (111)-oriented perovskites are little explored for photovoltaic applications, as they have highly distorted structures or strong excitonic natures [44-46]. For the ACI perovskites, this new entry of 2D structure with the formula $(G A) A_{n} M_{n} X_{3 n+1}$ (where $\mathrm{GA}=$ guanidinium) have a unique structure, as the smaller A cation both accommodates in the inorganic slabs and within the interlayer with the GA cation [41, 47]. Although the efficiency of $18.48 \%$ for the device based on this system was the highest reported for 2D PSCs based on (100)-oriented analogs, the few cation other than GA could be used to form the ACI structure, which restricted the further development [48].

RP phase perovskites with the general formula of $\mathrm{A}_{2}^{\prime} \mathrm{A}_{n-1} \mathrm{~B}_{n} \mathrm{X}_{3 n+1}$ remain the most commonly investigated $2 \mathrm{D}$ perovskite system, and have spacer layers that contain two offset monovalent organic cations between the inorganic frameworks. The adjacent unit cells are stacked together via van der Waals interactions, and present a staggered configuration along the $a b$-plane [22]. A wide variety of aliphatic or aromatic alkyl ammonium cations are currently being explored to extend the performance and stability of RP phase PSCs. The optical bandgap and electronic properties of 2D perovskite based films and devices are largely determined by the thickness number ( $n$-value) of the perovskite inorganic layers. As the $n$-value of $2 \mathrm{D}$ perovskites increases, their electronic bands broaden, which weakens quantum confinement and generates a narrower bandgap [49]. The higher the $n$-value of a 2D perovskite, the closer its optoelectronic properties are to the 3D perovskite component, and the $n$-value of $3 \mathrm{D}$ perovskite can be seen as infinite. So far, the highest efficiencies reported for RP phase PSCs have generally been recorded in devices based on quasi-2D perovskites with $n=5$ [29-31]. However, the weak interconnection between adjacent spacer layers sacrifices the structural stability of such RP phase PSCs under environmental stresses, and creates a large quantum confinement effect that limits charge transport and separation.

Different from the RP phase 2D perovskites, the interlayer space of $\mathrm{DJ}$ phase $2 \mathrm{D}$ perovskites feature a diammonium organic cation, which are characterized by only one spacer layer per unit cell. In the crystallographic aspect, the DJ phase 2D perovskites with the formula $\mathrm{A}^{\prime}$ $\mathrm{A}_{n} \mathrm{~B}_{n} \mathrm{X}_{3 n+1}$ exhibit a relative $(1 / 2,0)$ shift of the perovskite layers along the $a b$-plane, or no shift at all, whereas RP phase perovskite layers generally exhibit a $(1 / 2,1 / 2)$ shift $[50,51]$. The interlayer diammonium cations form a hydrogen bond with both of their adjacent interlayers, which endows the inorganic slabs with strong electrostatic interactions in the stacking direction. This gives DJ phase 2D perovskites a more stable crystal structure than $\mathrm{RP}$ 
A

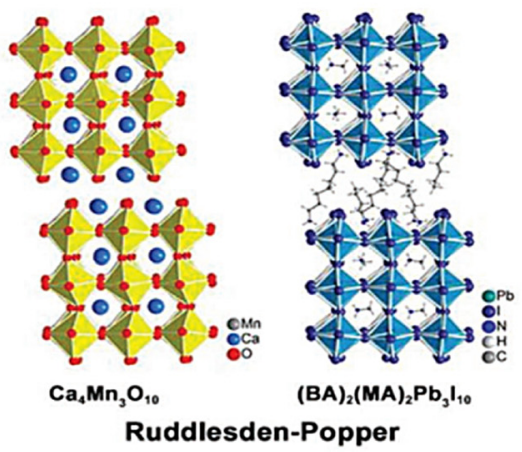

B

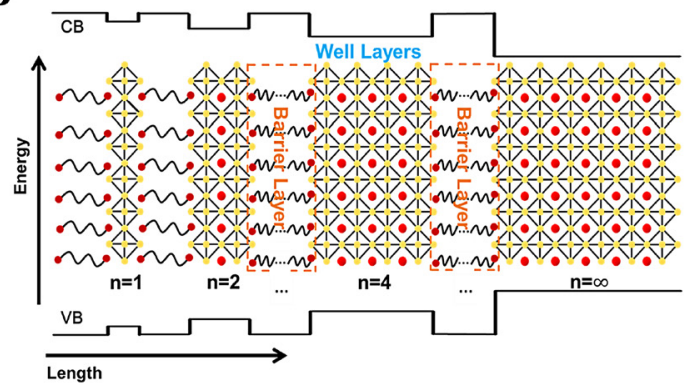

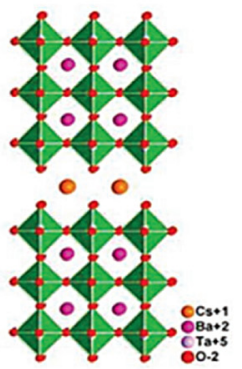

$\mathrm{CsBa}_{2} \mathrm{Ta}_{3} \mathrm{O}_{10}$ Dion-Jacobson

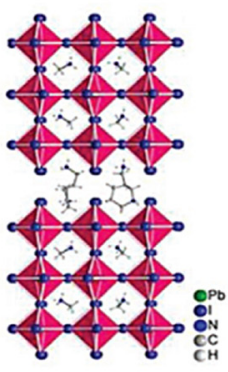

(3AMP)(MA) ${ }_{2} \mathrm{~Pb}_{3} \mathrm{I}_{10}$

C

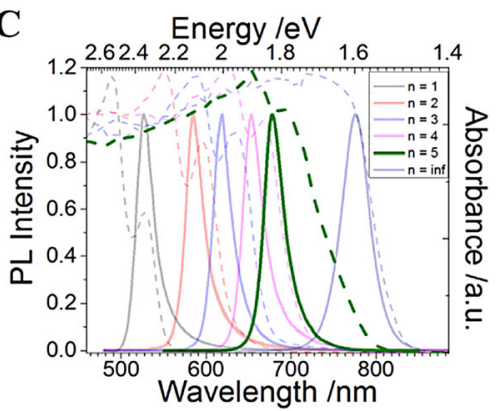

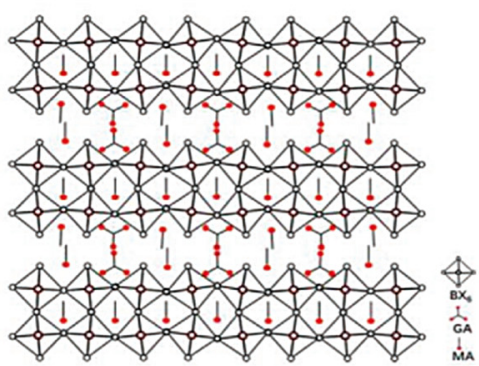

$(G A)_{2}(M A)_{2} B_{2} X_{r}$

ACI

D

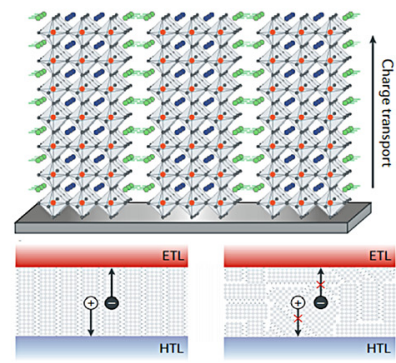

Figure 1: Crystal structure and basic optoelectronic properties of 2D perovskites.

(A) Comparison of crystal structures of RP-, DJ-, and ACl-phase 2D perovskites with $n=3$ phase. Reproduced with permission [22]. Copyright 2018, American Chemical Society Publications. (B) Schematic illustration of QW structure of DJ phase 2D perovskites containing QW barrier thickness, QW width and comparative band energy alignment. (C) Experimental optical absorption and emission spectra of 2D $(B A)_{2}(M A)_{n-1} \mathrm{~Pb}_{n} \mathrm{I}_{3 n+1}$ perovskites. Reproduced with permission [38]. Copyright 2018, Elsevier Inc. Publications. (D) Illustration of 2D perovskite with out-of-plane crystal arrangement. Under vertical stacking and regular QW distribution (left), charges can transport through the inorganic slabs and be collected by to charge transport layers. With a random phase distribution and horizontally oriented crystal arrangement (right), the insulted organic interlayer would restrict the charge transport pathway. Reproduced with permission [15]. Copyright 2018, Nature Publishing Group.

phase 2D perovskites, comprising a highly symmetric and uniform stacking of inorganic slabs that prevents interlayer displacement and enhances structural stability $[22,35]$.

Although 2D perovskites exhibit distinctive structural properties differed from their 3D counterparts, the low charge mobility, large exciton binding energy and optical bandgap severely hinder the practical exploitation in light harvesters. Solution-processed 2D perovskites have a natural QW structure, in which the electrically insulated organic layer serves as a barrier and the conducting inorganic framework acts as a well, as shown in Figure 1B [52]. The previous transient photoconductivity experiments conducted time-resolved terahertz spectroscopy have verified that charge transport preferentially occurs along the inorganic octahedral planes, and that excitons are bound in the multiple QWs [53]. In this scenario, quantum confinement along the direction vertical to the plane of inorganic slabs tends to be pronounced as $n$-values decrease, leading to the obvious blue-shifted exciton absorption peaks and sharp emission peaks across a wide range of wavelengths. Figure 1C illustrates the typical variations of absorption and emission spectra of $(\mathrm{BA})_{2}(\mathrm{MA})_{n-1} \mathrm{~Pb}_{n} \mathrm{I}_{3 n+1} 2 \mathrm{D}$ perovskites with the change of layer thickness.

The optical bandgap of 2D perovskites is also affected by the electronegativity of metal cations and the size of halide anions. If its bandgap is too large, the 2D perovskite may not function well as a light absorber, but the tunable absorption range of such materials makes them amenable for use in optoelectronic applications other than solar cells. On the other hand, the organic layers formed by the incorporated spacer ammonium barrier have a low dielectric constant of ca. 2.4, while the inorganic metal halide layers occupy a relatively higher dielectric constant of over 6 [52]. This dielectric-constant mismatch generates dielectric confinement, such that the inorganic layers are surrounded by spacer cations. The electric field generated by the charges in the high dielectric-constant material is largely unattenuated, due to the weak field-shielding effect of the low dielectric-constant material [54]. This strong dielectric confinement results in a large exciton binding energy (100$500 \mathrm{meV}$ ) in 2D perovskites, which can be tuned by varying the inorganic and organic components [38, 55-57]. 
Therefore, 2D perovskites typically exhibit strong electronhole pair interactions and thus a large barrier to charge transport across alternating inorganic sheets. The large binding energy in naturally formed QWs is closely associated with these dielectric and quantum confinement effects.

The confinement effect in such unique 2D structure also induces the anisotropic charge transport along various crystallographic directions, which are highly dependent on the crystal orientation (Figure 1D) [53, 58]. It was reported that the charge conductivity and mobility measured along the direction of inorganic slabs is higher than that of the perpendicular direction, which is restricted by the poor charge transportation within organic interlayers $[46,59]$. Therefore, achieving the out-of-plane crystal growth orientation is vital for 2D perovskites to ensure efficient charge transport and limit the radiative-recombination loss. In addition to the crystalline orientation, the chargetransfer behavior inside 2D perovskite films also exhibits a strong dependence on the thickness distribution of QWs. In previous research of 2D perovskites whether RP phase or DJ phase, a gradient phase distribution was detected along the direction from the top of film to the bottom. The holes could spontaneously transfer from high- $n$ phase to low- $n$ phase accompanied with electrons flowing in the opposite direction [60]. Hence, the rational control of the crystal orientation and ensuring a regular phase distribution enabled efficient charge transport across multiple QW structures and weakened the adverse effects caused by an insulating spacer layer [61]. In DJ phase 2D perovskites, the shortened interlayer distance weakens the quantumconfinement effect and lowers the barrier to charge transport between inorganic slabs. Moreover, DJ phase perovskites generally have a narrower thickness distribution of QWs than RP phase perovskites, which creates a more regular distribution of QW thickness and forms a flat energy landscape that accelerates charge separation and extraction [62, 63]. Such advantages contribute the DJ phase 2D perovskites to be a promising entry in photovoltaic application, rather than the single knowledge of such crystal structure in oxide perovskites. Overall, the distinct structural and optoelectronic properties of DJ phase 2D perovskites, together with their resistance to environmental damage, means that they have enormous potential for exploitation in various efficient room-temperature photovoltaic applications.

\section{Applications in solar cells}

The layered configurations consisted with bulky organic spacer cations and inorganic frameworks lead to the electronic quantum well (QW) structures of 2D perovskites. The existed QWs in 2D perovskites are capable of being exploited in light-emitting diodes for regulating electroluminescence properties or other optoelectronic applications. Undesirably, such structural properties may also induce the geometric effect and dielectric contrast between inorganic and organic layers, leading to the higher exciton binding energy of $2 \mathrm{D}$ perovskites against the 3D counterparts and the confinement to charge transport and extraction $[38,55]$. On the other hand, the presence of phase diversity is also a shortage to limit the device performance of 2D PSCs. Although the existed cascade energetic alignment in 2D perovskites ensure the charge transport through different $n$-value phase, it may lead to additional energy loss at the $p-n$ junctions, limiting the device parameters. Moreover, the solution-processed 2D perovskite films generally suffer from random crystal orientation, which hinders oriented charge transport and induces severe charge accumulation and recombination loss. Therefore, in addition to the intrinsic limitations of 2D perovskite materials, controlling the crystallization process to effectively manipulate the crystal orientation and phase distribution in solution-processed 2D perovskites is a key option to improve the device efficiency. Moreover, the organic spacer cations in 2D perovskites serve not only as insulating barriers for photogenerated charge carriers but also as dielectric moderators for the electrostatic interaction between electron-hole pairs. On the one hand, the appropriate design of spacer cations could guide the selfassembly process and achieve the preferred crystal orientation and phase purity. Besides, the spatial configurations of spacer cations also influence the electrostatic interaction force, improve the excitonic features and reduce the exciton binding energy [33]. Third, properly increasing the stacking-thickness number of symmetrical inorganic corner-sharing octahedral layers within 2D perovskites is an effective strategy for weakening the dielectricconfinement effect and broadening their electronic bands to reduce quantum confinement in naturally formed QW structures. However, how to synergistically keep the 2D perovskite structure, and alleviate the lattice distortion and QW random distribution under the varied incorporation concentration of spacer cations is still a problem worthy of being studied. Positively, broadening the application scope of 2D perovskites in photovoltaic field, like the exploitation of 2D/3D hybrid perovskites, represents a promising strategy to balance the trade-off between efficiency and stability of PSCs.

Based on the above considerations, the current strategies to optimize the structural order and related device performance of $\mathrm{DJ}$ phase 2D perovskites can be categorized 
as follows. (1) Ligand chemistry engineering. Various organic spacer cations can be used to adjust the coupling between 2D perovskite sheets and the energy levels of organic layers. The chemical configuration, spatial arrangement and functional groups of organic cations determine the intrinsic properties of 2D perovskites, such as their crystal dimensions, electronic structure, orientation and distribution of QWs. (2) QW-thickness control. The determination of effects of the $\mathrm{A}^{\prime}$-to-A ratio in precursor solutions on the crystal dimensions, $\mathrm{QW}$ arrangement and phase purity of 2D perovskites enables the rational control of crystallization process. (3) Mixed-cation engineering. The use of multiple A (or A')-site cationic dopants can enable control of lattice distortion, leading to out-of-plane crystal orientations. (4) Additive engineering. Additives can assist QW growth and also tune the orientation, film quality and related charge dynamics of $2 \mathrm{D}$ perovskites. (5) Solvent engineering. This can be used to control crystallization and nucleation processes, thereby optimizing crystallization kinetics and film quality. (6) Advanced perovskite systems. Examples of these include $\mathrm{Pb}$-free and 2D/3D hybrid heterojunction perovskites; to search for a promising route to achieve an optimal balance of device stability and efficiency across a broad range of compositions. The performance and stability statistics of various DJ phase layered PSCs and related 2D/3D hybrid heterojunction PSCs are summarized in Tables 1 and 2.

It should be admitted that all of above mentioned improvement strategies are mainly a continuation from the previous attempts of RP phase perovskites. Different reports often refer to different optimizing methods or some combination of them. The certain optimizing idea may be effective for a certain component, while not universal. Except for the design and synthesis of functional spacer cations, the choice of suitable fabrication technology largely relays on continuous explorations to determine the related roles in a given 2D perovskite system. The mechanistic synergies between different strategies, and between their crystallization and nucleation processes, are little understood. This hinders the combination of different optimization strategies to effectively work together and guide the positive modification to obtain desired 2D perovskite system. Similarly, there is no systematic theory to guide the selection principles of functional additives. Detailed mechanistic studies are therefore required to obtain a deeper understanding of how hot-casting or solvent-processing routes affect crystal-growth processes. There is also an urgent need for a facile, efficient and general method for the control of crystal kinetics and oriented order in 2D perovskites that contain different chemical spacer cations, as this will enable the development of high-performance DJ phase 2D perovskite systems for PSCs and other optoelectronic applications.

\subsection{Ligand chemistry engineering}

Although a wide variety of alkylammonium and aromatic alkylammonium cations have been used in 2D perovskites, the ligand chemistry of bifunctional or multifunctional spacer cations, which may lead to a greater diversity of 2D perovskite materials, has yet to be fully explored. In addition, there is limited knowledge on how variations in spacer ligands affect the properties of perovskite films. Functionalized spacer cations have also been used to fabricate 2D PSCs. The spatial arrangement, chemical structure, size and functional groups of organic cations affect the properties of 2D perovskites, such as their crystal dimensions, electronic structure, and orientation and distribution of QWs. Structural factors also influence the charge dynamics, optoelectronic properties and ultimate performance of the resulting 2D perovskite based devices. The appropriate design of organic ligands therefore enables control of crystal orientation and phase purity, which facilitates the fabrication of high-performance 2D PSCs. The organic ligands that are currently used for DJ phase perovskites, and the corresponding PCEs of $n \leq 5$ components derived from these, are summarized in Figure 2.

Kanatzidis et al. first reported the use of DJ phase perovskites incorporating 3-(aminomethyl)piperidinium $\left(3 \mathrm{AMP}^{2+}\right)$ and 4-(aminomethyl)piperidinium $\left(4 \mathrm{AMP}^{2+}\right)$ spacer cations in photovoltaic applications [22]. A detailed crystallographic analysis indicated that the position of the $-\mathrm{CH}_{2} \mathrm{NH}_{3}{ }^{+}$group on the piperidine core of these organic cations could influence the perovskite crystal structure, due to changes in hydrogen-bonding modes and the perturbation of inorganic slab orientations. For example, the variation in hydrogen-bonding networks resulted in the $\mathrm{Pb}-\mathrm{I}-\mathrm{Pb}$ angles of the 3AMP-based DJ phase perovskites being larger than those of the 4AMP systems. Notably, it was found that the closer the $\mathrm{Pb}-\mathrm{I}-\mathrm{Pb}$ bond angles were to $180^{\circ}$, the greater the overlap between $\mathrm{Pb} s$ and I $p$ orbitals [85-87]. $\mathrm{Pb}-\mathrm{I}-\mathrm{Pb}$ bond angles in DJ phase structures are classified as equatorial if they lie along the inorganic plane, and axial if they lie in the direction of the stacking axis (Figure 3A); the former are more associated with bandgap width and the latter with excitonic behavior [88]. The $\mathrm{Pb}-$ $\mathrm{I}-\mathrm{Pb}$ bond angles of the $\mathrm{DJ}$ phase perovskites reported by Kanatzidis et al. were largely equatorial, which would have led to a strong anti-bonding interaction and thus a shallow valence-band maximum, and accounts for the narrower bandgap that was observed in the 3AMP-based perovskites 


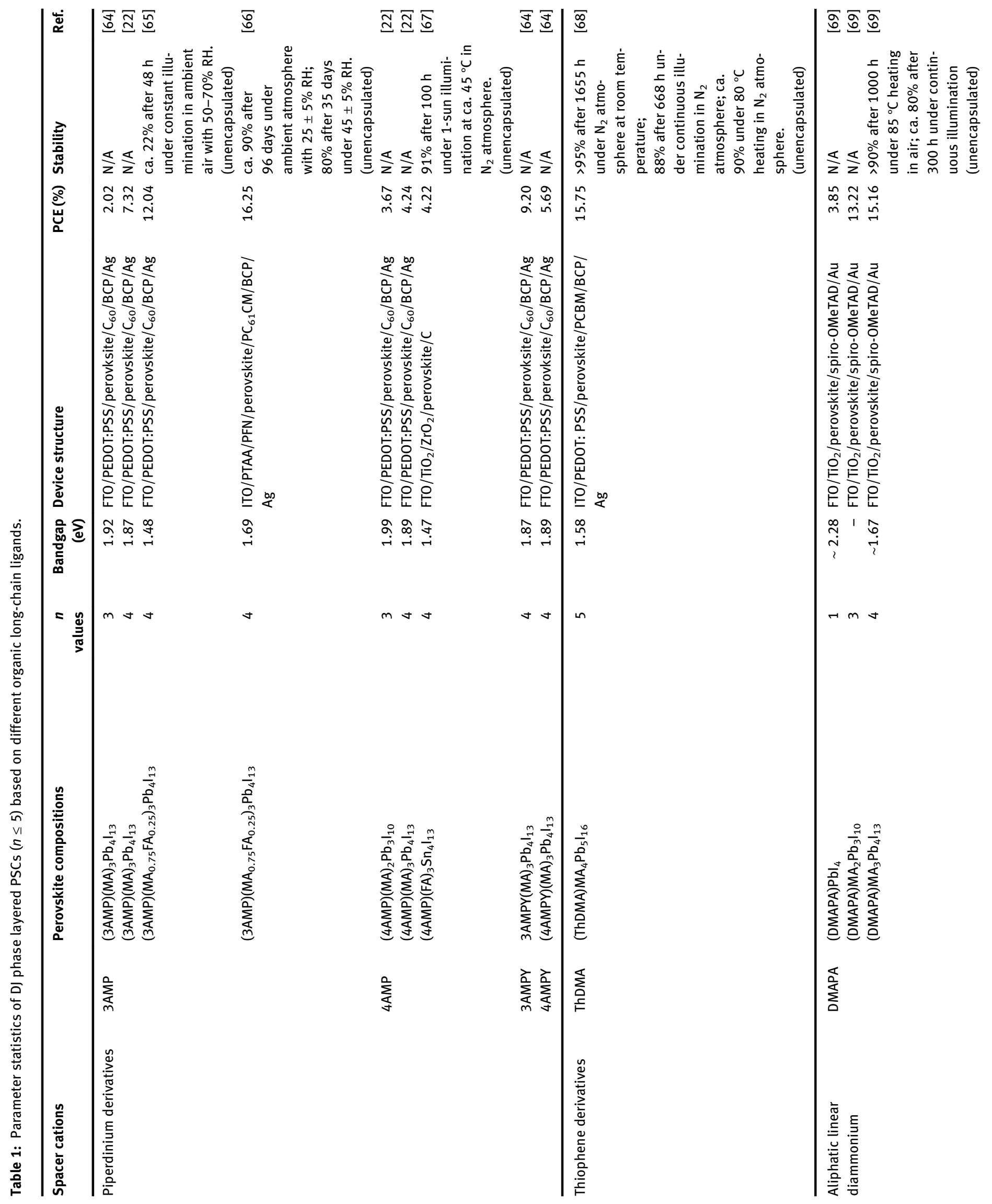




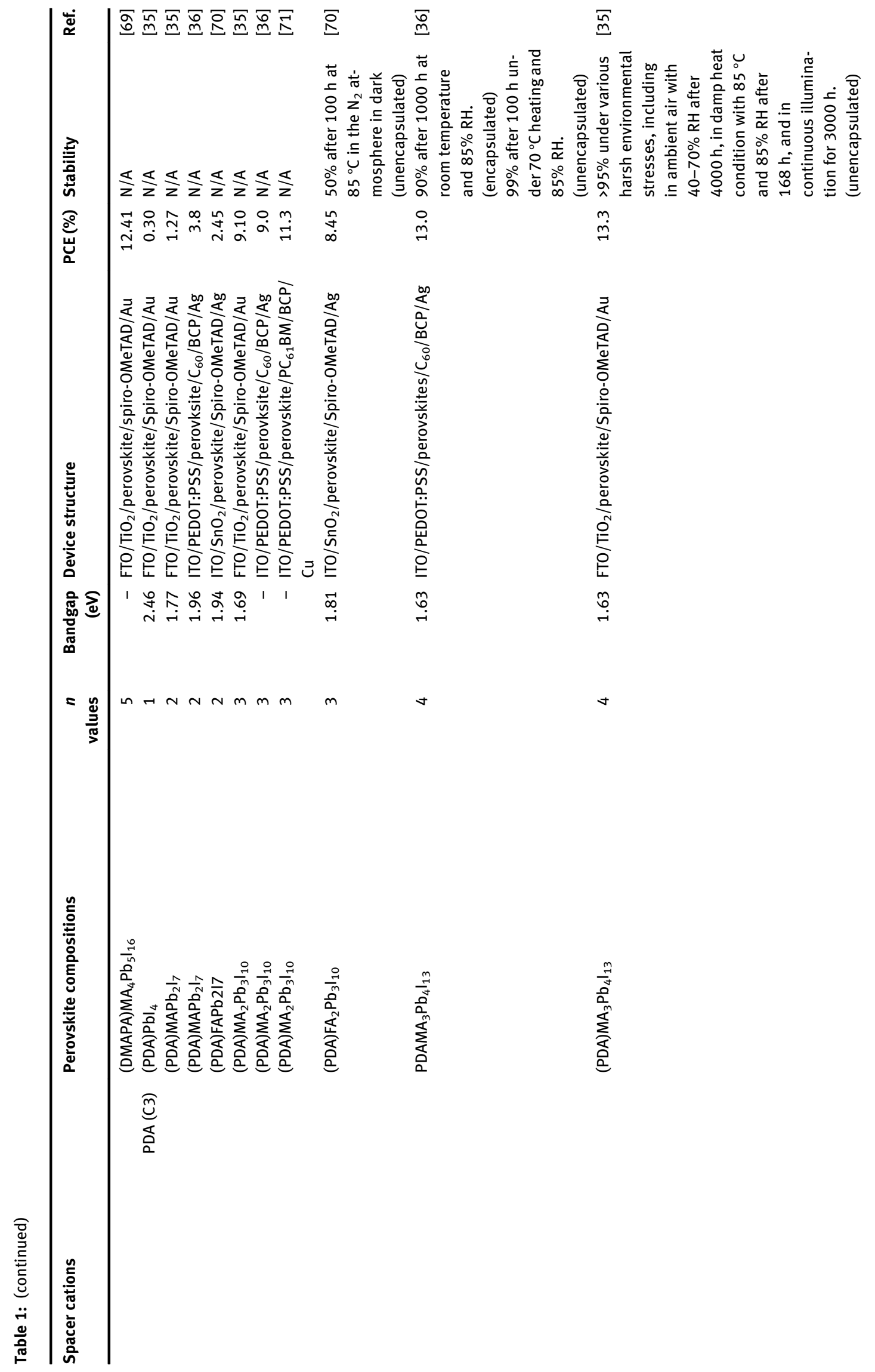




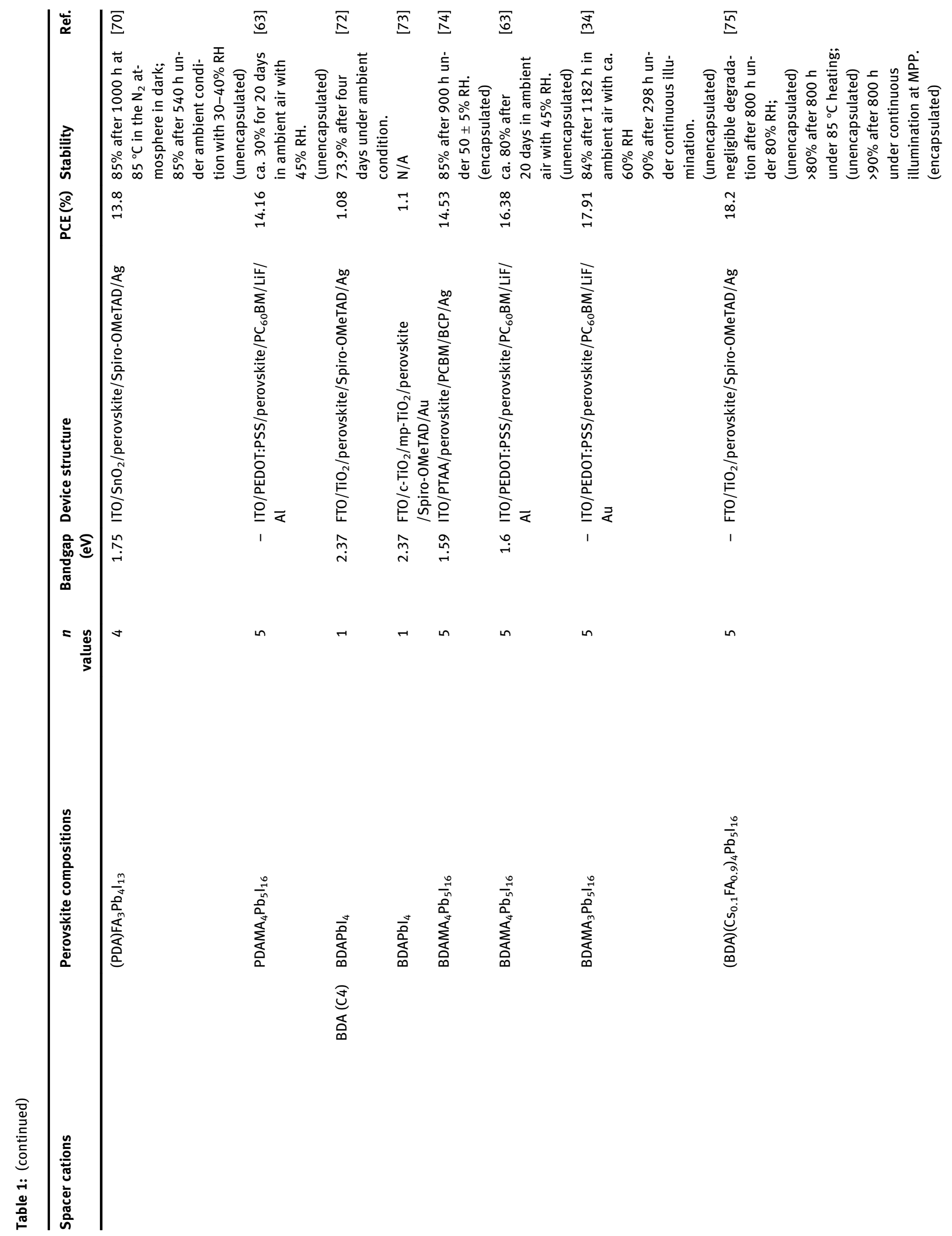




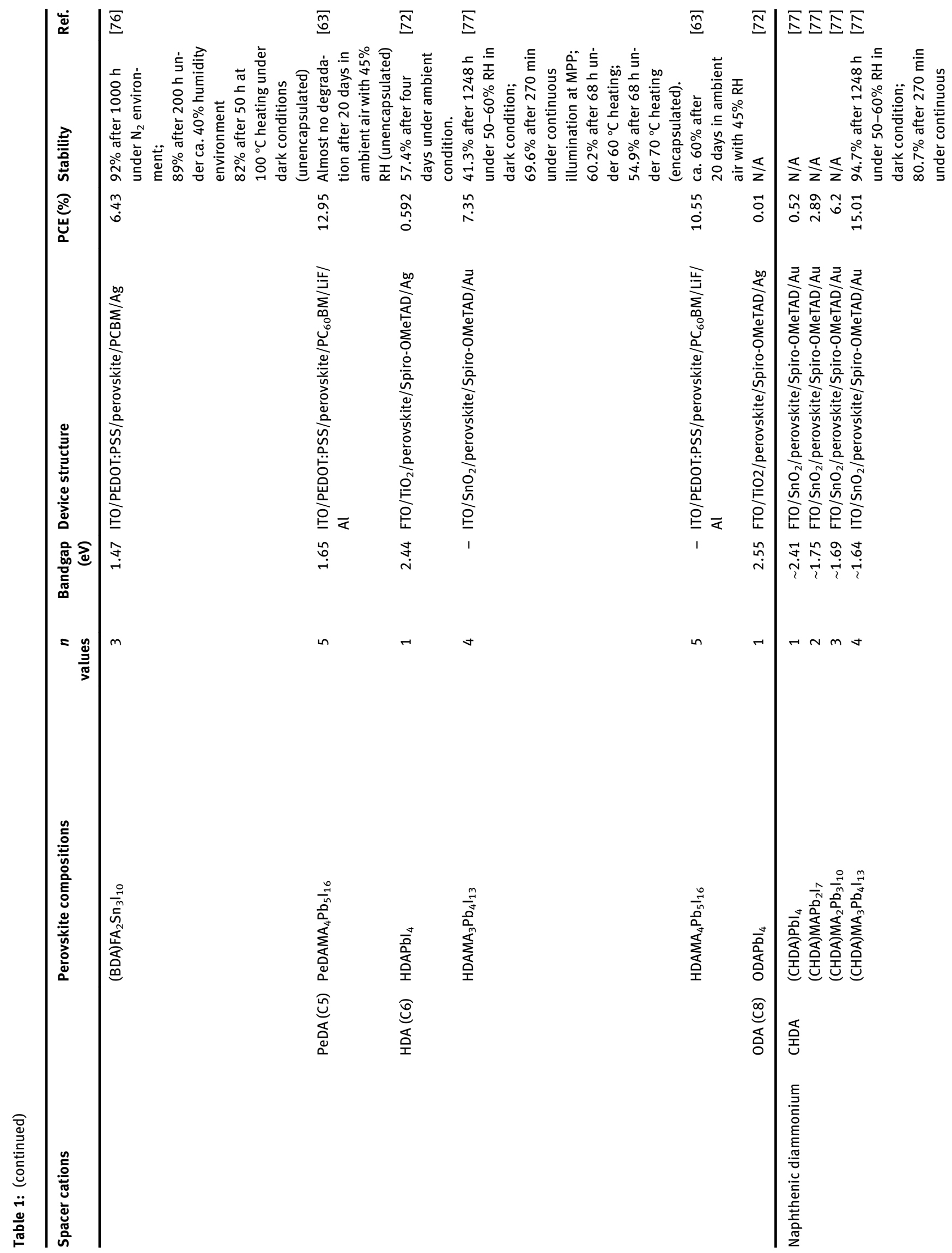




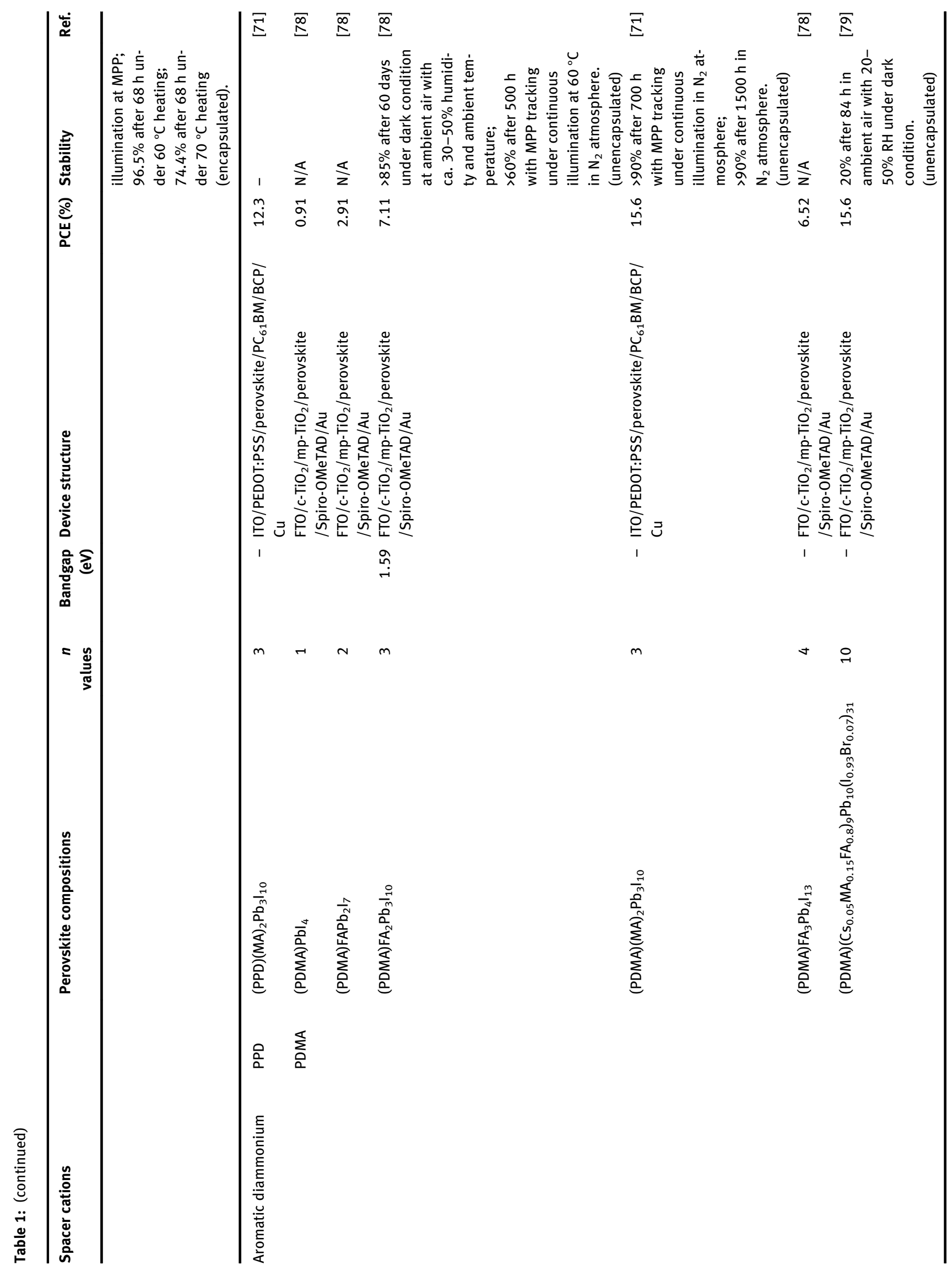


Table 2: Parameter statistics of 2D/3D hybrid heterojunction based on DJ phase perovskite.

\begin{tabular}{|c|c|c|c|c|}
\hline \multicolumn{5}{|l|}{ 2D/3D bulk heterojunction } \\
\hline Perovskite composition & Device structure & $\begin{array}{l}\text { PCE } \\
\text { (\%) }\end{array}$ & Stability & Ref. \\
\hline $\mathrm{Cs}_{0.15} \mathrm{FA}_{0.83} \mathrm{PDA}_{0.02} \mathrm{Pbl}_{3}$ & $\begin{array}{l}\text { ITO/Poly-TPD/perov- } \\
\text { skite/PCBM/BCP/Ag }\end{array}$ & 18.10 & $\begin{array}{l}82.8 \% \text { after } 500 \mathrm{~h} \text { under continuous illumi- } \\
\text { nation in ambient air at } 60^{\circ} \mathrm{C} . \\
\text { (encapsulated) }\end{array}$ & {$[80]$} \\
\hline$\left(\mathrm{EDBEPbl}_{4}\right)_{0.03}\left(\left(\mathrm{Cs}_{0.05}\left(\mathrm{FA}_{0.83} \mathrm{MA}_{0.17}\right)_{0.95} \mathrm{~Pb}\left(\mathrm{I}_{0.83} \mathrm{Br}_{0.17}\right)_{3}\right)_{0.97}\right.$ & $\begin{array}{l}\mathrm{FTO} / \mathrm{SnO}_{2} / \text { perov- } \\
\text { skite/Spiro-OMeTAD/ } \\
\mathrm{Au}\end{array}$ & 21.06 & $\begin{array}{l}>90 \% \text { after } 3000 \mathrm{~h} \text { under ambient atmo- } \\
\text { spheric conditions } \\
>90 \% \text { after } 1000 \mathrm{~h} \text { at } 65 \% \text { relative humidity } \\
>95 \% \text { after } 500 \mathrm{~h} \text { under continuous illumi- } \\
\text { nation at MPP. (encapsulated) }\end{array}$ & {$[81]$} \\
\hline$\left(M_{0.5} \mathrm{FA}_{0.5}\right)_{3}\left(\mathrm{~Pb}_{0.5} \mathrm{Sn}_{0.5}\right)_{4} \mathrm{I}_{13}+2.5 \mathrm{~mol} \% 3 \mathrm{AMP}$ & $\begin{array}{l}\text { ITO/PEDOT:PSS/ } \\
\text { perovskite/PCBM/ } \\
\mathrm{BCP} / \mathrm{Ag}\end{array}$ & 20.09 & $\begin{array}{l}73.2 \% \text { after } 100 \mathrm{~h} \text { under continuous illumi- } \\
\text { nation in ambient air with } 20-50 \% \mathrm{RH} \text { at } \\
\text { room temperature. (encapsulated) }\end{array}$ & {$[82]$} \\
\hline \multicolumn{5}{|l|}{ 2D/3D hierarchical heterojunction } \\
\hline $\mathrm{HDAPbl}_{4} / \mathrm{Cs}_{0.05}\left(\mathrm{MA}_{0.17} \mathrm{FA}_{0.83}\right)_{0.95} \mathrm{~Pb}\left(\mathrm{I}_{0.83} \mathrm{Br}_{0.17}\right)_{3}$ & $\begin{array}{l}\mathrm{FTO} / \mathrm{SnO}_{2} / \text { perov- } \\
\text { skite/Spiro-OMeTAD/ } \\
\mathrm{Ag}\end{array}$ & 20.31 & $\begin{array}{l}80 \% \text { after } 60 \text { days under } 55-75 \% \text { RH at } \\
\text { temperature of ca. } 25-40{ }^{\circ} \mathrm{C} \text {; } \\
60 \% \text { after } 350 \mathrm{~h} \text { at } 85^{\circ} \mathrm{C} \text { heating. } \\
\text { (unencapsulated) }\end{array}$ & {$[83]$} \\
\hline $\mathrm{ODAPbl}_{4} /\left(\mathrm{FA}_{0.85} \mathrm{MA}_{0.15}\right) \mathrm{Pb}\left(\mathrm{I}_{0.85} \mathrm{Br}_{0.15}\right)_{3}$ & $\begin{array}{l}\mathrm{FTO} / \mathrm{c}-\mathrm{TiO}_{2} / \mathrm{mp}^{-\mathrm{TiO}_{2}} / \\
\text { perovskite/Spiro- } \\
\text { OMeTAD/Au }\end{array}$ & 21.6 & $\begin{array}{l}90 \% \text { after } 360 \mathrm{~h} \text { under } 85 \% \mathrm{RH} \text { at room } \\
\text { temperature. }\end{array}$ & [84] \\
\hline
\end{tabular}

$\mathbf{A}$<smiles>CCC1CCCCC1</smiles><smiles>C=CC1CCCCC1</smiles><smiles>O=Cc1ccccc1CO</smiles>
उAMP 3 ЗАMPY

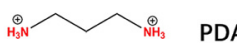

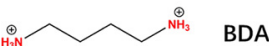

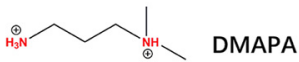

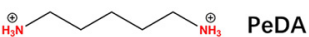<smiles>OCCCCCCCCCCCC[Hg]</smiles>

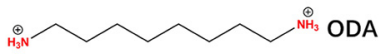

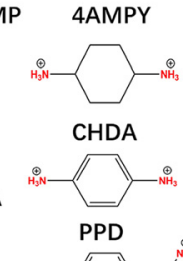

$$
\text { PDMA }
$$

B

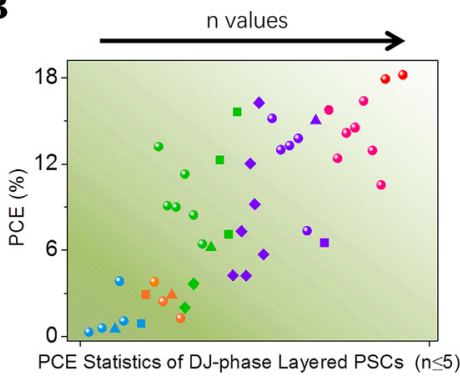<smiles>CCCc1ccc(CO)s1</smiles>

ThDMA

Ref.

\section{[}

.

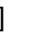


A

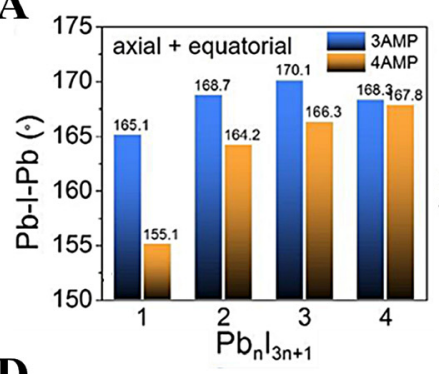

D

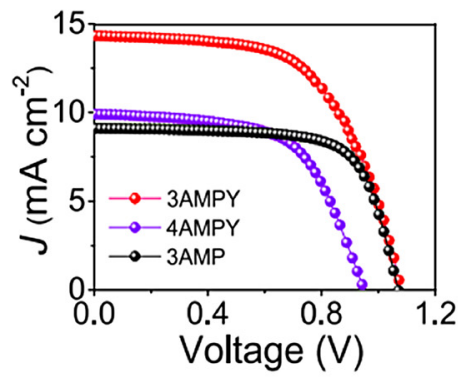

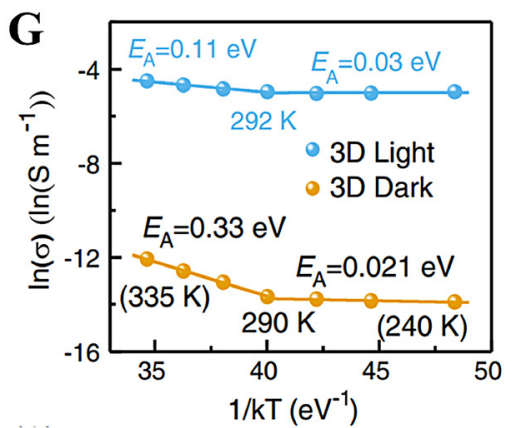

B

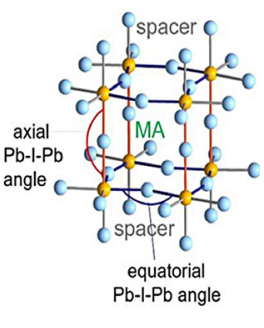

$\mathbf{E}$

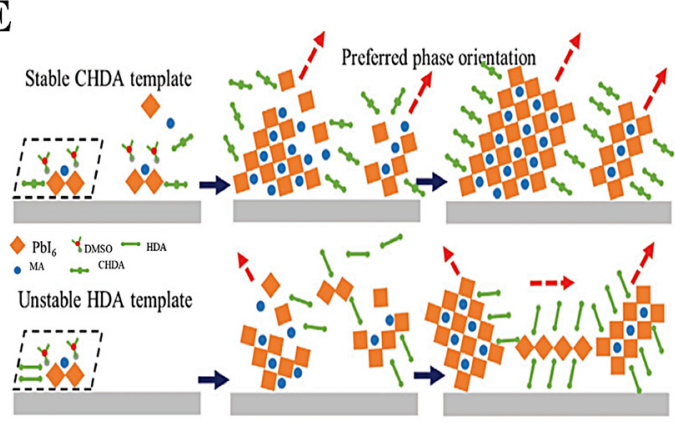

C

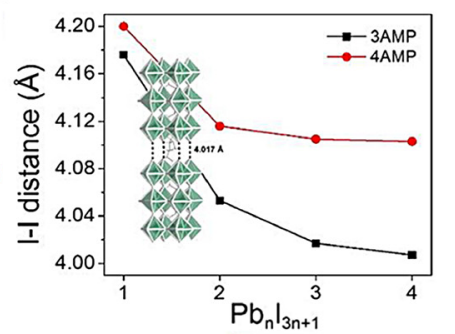

F

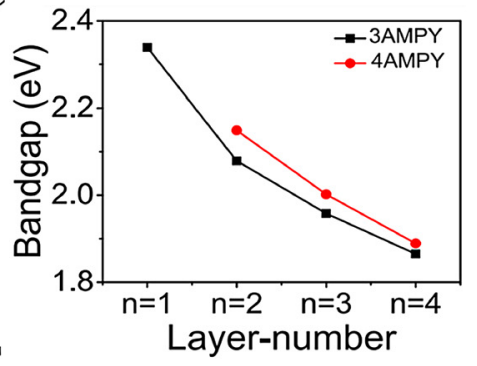

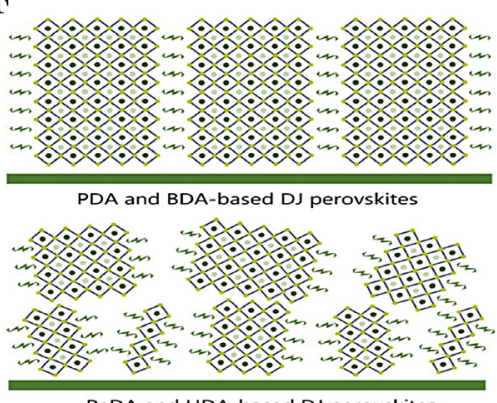

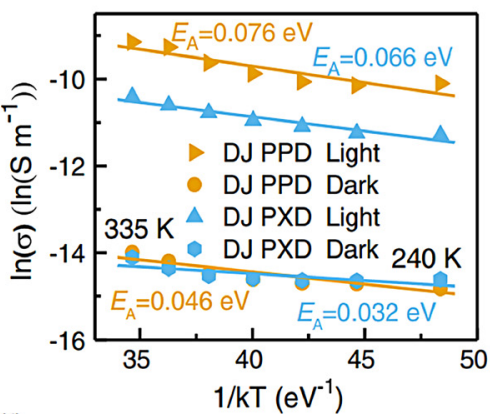

H

PeDA and HDA-based DJ perovskites

Heat stability @ $85^{\circ} \mathrm{C}$ and $85 \%$ RH

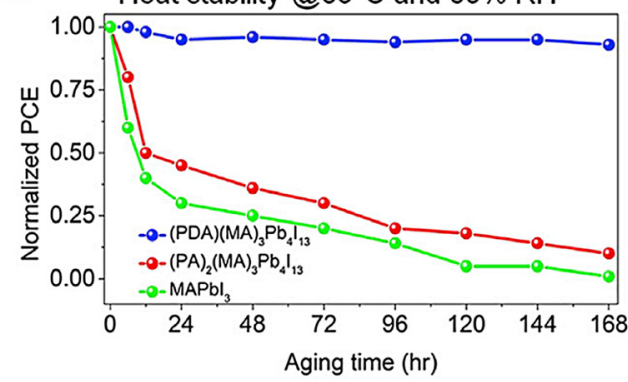

Figure 3: Ligand chemistry modification in DJ phase perovskites.

(A) Average axial and equatorial angles of 3AMP- and 4AMP-based DJ series of layered perovskites $(n=1-4)$ and the definitions of the respective axial and equatorial $\mathrm{Pb}-\mathrm{I}-\mathrm{Pb}$ angles. (B) $\mathrm{I} \cdots \mathrm{I}$ distance trend in 3AMP- and 4AMP-based $\mathrm{DJ}$ phase 2D perovskites, where 3AMP series present closer distance. Reproduced with permission [22]. Copyright 2018, American Chemical Society Publications. (C) Comparison of bandgaps for $3 A M P Y$ - and $4 A M P Y$-based perovskite series. (D) $J-V$ curves for the champion PSCs using (3AMPY) $(M A)_{3} P b_{4} I_{13}$,

(4AMPY)(MA) ${ }_{3} \mathrm{~Pb}_{4} \mathrm{I}_{13}$, and (3AMP)(MA) ${ }_{3} \mathrm{~Pb}_{4} \mathrm{l}_{13}$ compositions. Reproduced with permission [64]. Copyright 2019, American Chemical Society Publications. (E) Schematic of crystallinity kinetics for (CHDA)MA $\mathrm{Pb}_{4} \mathrm{I}_{13}$ and ( $\left.\mathrm{HDA}\right) \mathrm{MA}_{3} \mathrm{~Pb}_{4} \mathrm{I}_{13}$ films, showing the different crystal orientations. Reproduced with permission [77]. Copyright 2020, Wiley-VCH Publications. (F) Schematic diagram of the homogeneous and random QW distribution of DJ phase perovskite films based on different alkyl chain lengths of linear organic ligands. Reproduced with permission [63]. Copyright 2019, Wiley-VCH Publications. (G) Temperature-dependent conductivity measurement results of 3D $\mathrm{MAPbl}_{3}$ (left) films along with the PPD- and PXD-based DJ phase 2D perovskite $(n=3)$ films (right). Reproduced with permission [71]. Copyright 2020, Wiley-VCH Publications. (H) Stability test results of $\mathrm{MAPbl}_{3},(\mathrm{PA})_{2}(\mathrm{MA})_{3} \mathrm{~Pb}_{4} \mathrm{I}_{13}$, and $(\mathrm{PDA})(\mathrm{MA})_{3} \mathrm{~Pb}_{4} \mathrm{I}_{13}$ based devices under damp heat stress with $85^{\circ} \mathrm{C}$ and $\left.85 \% \mathrm{RH}\right)$. Reproduced with permission [35]. Copyright 2018, Elsevier Inc. Publications.

than in their 4AMP analogs. In addition, as the layer thickness increases, the perturbation of the inorganic slabs by a spacer cation dramatically diminishes and the I $\cdots I$ distance gradually decreases (Figure 3B). The gradual increase in the $\mathrm{Pb}-\mathrm{I}-\mathrm{Pb}$ angles as the $n$-values increased from 1 to 4 would have directly affected the optoelectronic properties of these materials. Moreover, these DJ phase perovskites have narrower bandgaps and much shorter I $\cdots \mathrm{I}$ interlayer distances than their RP and ACI phase analogs, indicating the greater potential of $\mathrm{DJ}$ phase perovskites for photovoltaic applications [22]. The relationships between spacer cation structure, electrostatic attraction and electronic band structure were further verified by electronic structure calculations. Under a planar $p$-i-n device structure of FTO/PEDOT:PSS/perovskite/ $\mathrm{C}_{60} / \mathrm{BCP} / \mathrm{Ag}$, Kanatzidis et al. fabricated the devices containing (3AMP)(MA) $)_{3} \mathrm{~Pb}_{4} \mathrm{I}_{13}$ component $(n=4)$ delivered a relatively higher PCE of $7.32 \%$ than the corresponding 4AMP$\mathrm{MA}_{3} \mathrm{~Pb}_{4} \mathrm{I}_{13}$ based devices (4.24\%). The better performance of the 3AMP-based device was attributed to its narrower 
optical bandgap and enhanced charge mobility relative to the 4AMP-based case, which was due to increased band dispersion [22]. The authors also emphasized that organic spacer cations have an array of effects on the optical properties of inorganic layers, as the size and shape of bulky cations determine their steric effects, their charge determines the electrostatic attraction effects and their distribution of functional groups determines their hydrogen-bonding and band-dispersion forces.

A subsequent study by Kanatzidis group further validated the effects of a variety of cyclic diammonium cations possessing a range of molecular conformations and conjugated groups on the properties of inorganic slabs in DJ perovskite materials [64]. The authors synthesized pyridinium analogs of their original piperidinium cations, namely 3-(aminomethyl)pyridinium (3AMPY) and 4-(aminomethyl)pyridinium (4AMPY), and used these to construct the DJ phase perovskites. The aromatic pyridinium rings in the AMPY cations were more rigid than the aliphatic piperidinium rings in the AMP cations, and the delocalized $\pi$-electron cloud in the AMPY cations led to the AMPY-based perovskites having greater dielectric constants of ca. 3 than the AMP-based perovskites with value of ca. 2. This decreased the dielectric mismatch between the inorganic slabs and barrier layers in the formed QWs, and improved the corresponding dielectric confinement effect in the resultant systems. The smaller exciton-binding energy of these aromatic systems gave them much more efficient charge separation and transport than their aliphatic analogs. The high symmetry of the 4AMPY cation meant that it maintained the correct DJ phase structure under $(0,0)$ displacements. In contrast, the 3AMPY perovskite structures were slightly offset, due to the structural perturbation induced by the positions of functional groups in spacer ligands. To elucidate the effect of structure on the optical properties of the 3AMPY perovskites, Kanatzidis et al. studied the relationship between the extent of penetration of the positively charged $\left(\mathrm{NH}_{3}{ }^{+}\right)$ ends of ligands, the strength of the electrostatic interaction between $\mathrm{NH}_{3}{ }^{+}$and terminal $\mathrm{I}^{-}$, the octahedral distortion and the orbital overlap/optical bandgap [64]. It was found that the greater the penetration of the ammonium cation, the greater the distance between the $\mathrm{NH}_{3}{ }^{+}$and the $\mathrm{I}^{-}$and the weaker the electronic interactions and hydrogen bonding. The $\mathrm{Pb}-\mathrm{I}-\mathrm{Pb}$ angles were thus greater, which decreases the distortion of the inorganic octahedral structure, thereby increasing the overlap of the $\mathrm{Pb} s$ and $\mathrm{I} p$ orbitals and generating smaller bandgaps in the 3AMPY-based perovskites (Figure 3C).

The structural distortion induced by the increased $\mathrm{Pb}-$ $\mathrm{I}-\mathrm{Pb}$ angles also influenced the optical properties of the corresponding perovskite devices. In some examples, the smaller $\mathrm{Pb}-\mathrm{I}-\mathrm{Pb}$ angles in the more distorted 4AMP-containing structures offseted the bandgap decrease that resulted from the narrower interlayer spacing, which resulted in the overall bandgaps of $4 \mathrm{AMPY}$ and 4AMP systems being much closer to that of butylammonium (BA) systems with the same layer number $(n=4)$. A similar trend was seen in compounds based on linear symmetric diammonium cations. However, despite the large equatorial $\mathrm{Pb}-\mathrm{I}-\mathrm{Pb}$ angles in $\left(\mathrm{NH}_{3} \mathrm{C}_{8} \mathrm{H}_{16} \mathrm{NH}_{3}\right)\left(\mathrm{CH}_{3} \mathrm{NH}_{3}\right)_{2} \mathrm{~Pb}_{3} \mathrm{I}_{10}$, the 4AMP system with the same layer number $(n=3)$ had a smaller bandgap, due to its narrower interlayer spacing [88]. In terms of device performance, those containing (3AMPY)(MA) ${ }_{3} \mathrm{~Pb}_{4} \mathrm{I}_{13}$ exhibited an excellent PCE of $9.2 \%$, which was attributed to their improved film crystallinity and vertical crystal orientation, and extended photoluminescence (PL) lifetime. In contrast, the best PCEs of devices composed of (4AMPY)($\mathrm{MA})_{3} \mathrm{~Pb}_{4} \mathrm{I}_{13}$ or (3AMP)(MA) $)_{3} \mathrm{~Pb}_{4} \mathrm{I}_{13}$ were relatively low (5.69 and $6.89 \%$, respectively) (Figure 3D) [64].

Besides the piperidinium analogues, the cyclic diammonium cations based on thiophene units have also been used in DJ phase perovskites. Liu et al. synthesized a thiophene derivative, 2,5-thiophenedimethylammonium iodide (ThDMAI), and incorporated this into DJ phase 2D (ThDMA)MA ${ }_{4} \mathrm{~Pb}_{5} \mathrm{I}_{16}$ PSCs. It was found that the inclusion of a certain amount of dimethylsulfoxide (DMSO) in the precursor solution resulted in a strong coordination interaction between DMSO and $\mathrm{PbI}_{2}$, which controlled crystal growth and orientation, leading to good crystallinity and well-orientated crystals in the resulting 2D perovskite film. This facilitated fast charge transport and suppressed nonradiative recombination loss. Thus, the corresponding device delivered a high efficiency of $15.75 \%$ and excellent long-term stability, demonstrating the utility of a thiophene-based spacer cation combined with device engineering. The crystallization kinetics, crystal orientations and phase distributions of 2D perovskites are closely related to the structures of their spacer cations. For example, $\mathrm{Xu}$ and coworkers used a novel organic ligand, trans-1,4-cyclohexanediamine (CHDA), for the construction of DJ phase 2D perovskites. It was observed that the tilted crystal orientation and phase alignment of (CHDA) $\mathrm{MA}_{3} \mathrm{~Pb}_{4} \mathrm{I}_{3}$ perovskite film was due to a spontaneous selfalignment process, as shown in Figure 3E [77]. In contrast, the perovskite film based on the CHDA-isomer 1,6-diaminohexane (HDA) suffered from unstable intermediate phases, and in response to thermal heating developed random orientations and a graded distribution of crystals, with low $n$-value QWs close to the bottom of the film and high $n$-value QWs at the surface. The optimized charge 
transfer and crystal quality of the CHDA-based DJ phase 2D perovskite led to a high device efficiency of up to $15.01 \%$, and greater device stability under various harsh environmental conditions, such as moisture, heat and illumination.

In addition to the rigid skeleton of benzene-based aromatic diammonium cations leading to more organized structures than those based on aliphatic species, the free $\pi$-electrons of the aromatic core can also enhance the charge transport properties. Thus, to explore the effect of ligand chemistry on the structural and optoelectronic properties of perovskite films, Gratzel et al. used 1,4-phenylenedimethanammonium (PDMA) as a spacer ligand in FA-based DJ phase perovskites with a $n$-values ranging from 1 to 4 [78]. The resulting rigid 2D backbone interacted with the $\pi-\pi$ stacking within the PDMA-based interlayer to enhance the material crystallinity. The flexible methylamine linkers largely prevented geometrical mismatches and maintained a robust overall structural framework. Aside from a negative relationship between the size of the optical bandgap and the numbers of inorganic slabs, it was found that higher $n$-values had greater effects on crystallite size and morphology, and crystalline orientation in the resulting films. Moreover, similar to the RP phase perovskite systems, edge states were found to have an effect in DJ phase layered perovskites. Thus, in $n \geq 2$ compositions, red-shifted PL signals with higher emission intensities and longer exciton lifetimes were observed in addition to the main exciton signals. The large Stokes shifts were due to internal exciton dissociation from the photogenerated excitons to free carriers that were presumably trapped at the edges, and thus protected from the nonradiative decay process [89]. In terms of device performance, (PDMA)FA ${ }_{2} \mathrm{~Pb}_{3} \mathrm{I}_{10}$-based PSCs with a conventional mesoscopic structure devoid of additives or antisolvent modification achieved a PCE of $7.11 \%$ and a good shelf-life stability. Etgar et al. also used the PDMA as the spacer cation, and increased device efficiency up to $15.6 \%$ by optimizing $n$-values to 10 and combining triple A-site cations and mixed X-site halogen ions [79]. The additional electrostatic potential in the DJ phase perovskite increased the standard buildup in potential and increased the overall voltage of the corresponding devices. The good band alignment between the layered perovskite and selective contacts improved the charge-collection efficiency and recombination lifetime compared to the analogous 3D perovskite. The accumulation of carriers at the aromatic core prolonged the electron recombination lifetime and diffusion coefficient, creating a long diffusion length of about $800-1100 \mathrm{~nm}$, compared to ca. $800 \mathrm{~nm}$ in the 3D perovskite. The facile deposition process led to PDMA-based DJ phase perovskites with good film quality, and was less sensitive to experimental conditions than more complex fabrication methods, which ensured desirable device repeatability. It is worth mentioning that the $d$-spacing between the inorganic frameworks within a (PDMA) $\mathrm{Pb}\left(\mathrm{I}_{0.93} \mathrm{Br}_{0.07}\right)_{4}$ film $(n=1)$ was determined to be $\sim 11.1 \AA$, which was even larger than that in traditional RP phase perovskites, such as ca. $7 \AA$ in a BA-based system [36].

In other work, Yuan et al. compared the ion-migration behavior of $p$-phenylenediamine (PPD) and $p$-xylylenediamine (PXD)-based DJ phase perovskites $(n=3)$, and found that the transition temperature from electronic conductivity to ionic conductivity was up to $335 \mathrm{~K}$ in two cases, but did not present the obvious difference in transition temperature between each other (Figure 3G) [71]. Given the low transition temperature of $290 \mathrm{~K}$ for the 3D $\mathrm{MAPbI}_{3}$ perovskite, this showed that ion migration could be efficiently constrained in DJ phase perovskites, which led to the negligible efficiency hysteresis and long-term operational stability of the corresponding devices. In addition, the different interlayer distance resulting from the variation of spacer cations appeared to have a negligible effect on to the final ion conductivity, so did the possible existed 3D-like phase in DJ phase perovskites.

Currently, DJ phase layered PSCs based on the linear alkyl diammonium cations still occupy the record efficiencies of ca. $18 \%(n \leq 5)$. However, it seems to more empirically category such ditopic organic cations based 2D metal halide perovskites into DJ phase structure. The detailed structural information characterized by single crystal data is still limited. Lemmerer and Billing found that $\left(\mathrm{NH}_{3} \mathrm{C}_{m} \mathrm{H}_{2 m} \mathrm{NH}_{3}\right)$ $\mathrm{PbI}_{4}$ perovskites, in which the linear diamines with certain number of carbon-chain (such as $m=4,8,10,12$ ) could form 2D perovskite configurations, whereas those with $m=5$ or 7 formed lower-dimensionality structures [90]. Gardner et al. presented detailed crystallographic analyses of $\mathrm{BDAPbI}_{4}$ ( $m=4$, 1.4-butanediammonium abbreviated as BDA), $\mathrm{HDAPbI}_{4}(m=6$, 1.6-hexamethylenediammonium abbreviated as $\mathrm{HDA})$ and $\mathrm{ODAPbI}_{4}(m=8$, abbreviated as ODA) perovskites revealing that compared to the cubic $\mathrm{MAPbI}_{3}$ structure, there was significantly reduced order in the intraplanar inorganic layers of these materials, together with smaller $\mathrm{Pb}-\mathrm{I}-\mathrm{Pb}$ angles [72]. Kanatzidis and coworkers used single-crystal $\mathrm{X}$-ray characterization to reveal that $\left(\mathrm{NH}_{3} \mathrm{C}_{m} \mathrm{H}_{2 m} \mathrm{NH}_{3}\right)\left(\mathrm{CH}_{3} \mathrm{NH}_{3}\right)_{n-1} \mathrm{~Pb}_{n} \mathrm{I}_{3 n+1}$ perovskites in which $m=8$ and 9 had an RP phase structure, which was consistent with their optical properties. The linear organic ligands in these perovskites were too long to maintain QW rigidity across the entire structure, and thus had a folded conformation. However, the inorganic slabs in these layered perovskites tended to adopt a staggered configuration in which 
each layer was offset by half a $\left[\mathrm{PbI}_{6}\right]^{4-}$ octahedron, which was distinct from the stretched conformation of alkyl-chain ligands and the interdigitating motif of spacer cations in RP-type perovskites [88]. The authors also evaluated the crystal orientation and device stability of DJ phase perovskite films by using straight alkyl-chain ligands of varied lengths. It was found that with the same solvent components, $\left(\mathrm{NH}_{3} \mathrm{C}_{m} \mathrm{H}_{2 m} \mathrm{NH}_{3}\right)\left(\mathrm{CH}_{3} \mathrm{NH}_{3}\right)_{2} \mathrm{~Pb}_{3} \mathrm{I}_{10}$ films with $m=8$ and 9 exhibited discrete Bragg spots in grazing-incidence wideangle X-ray scattering (GIWAXS) patterns, indicating that they had a strong, near-single-crystal crystalline orientation. However, analogous films with $m=4,6$ or 7 exhibited near-random orientations and polycrystalline properties. Further detailed comparisons revealed the coexistence of parallel and perpendicular orientations in a film with $m=9$, while the inorganic slabs in a film with $m=8$ only had highly vertical alignments against the substrate. A certain QW orientation was critical to achieve efficient charge transport and extraction from the absorber layer to the charge transport layer, and to enable escape from traps via insulating layers. Moreover, the film with $m=8$ had the best thermal, light and air stability of all of the films with $n=3$ and that contained different alkyl diammonium ligands, indicating its good potential for device fabrication.

An examination of the development process of DJ phase PSCs using linear diammonium cations shows that studies have focused on short-chain ligands with $m=3$ or 4 for application in high-performance devices. Gardner et al. first used the layered alkyl diammonium-based $\mathrm{BDAPbI}_{4}$ perovskite in solar cells, and these achieved a relatively low PCE of $1.08 \%$ when fabricated in humidity conditions [72]. Then, the propane-1,3-diammonium (PDA) cation was used instead of the BA cation in layered perovskites to reduce the interlayer distance (from $7 \AA$ with BA to $2 \AA$ with PDA) and improve the quantum confinement between the highly conductive inorganic slabs and insulating spacer layers. This resulting (PDA) $\mathrm{MA}_{3} \mathrm{~Pb}_{4} \mathrm{I}_{13}$ perovskite displayed improved electrical conductivity and decreased recombination loss, leading to a PCE of $13.0 \%$ in the corresponding devices. Notably, the 2 A distance between the inorganic sheets in this PDA-based system was shorter than the van der Waals distance ( $4 \AA$ ), which resulted in a strong interaction between neighboring units that increased device stability. The PDA-based device also maintained 90 and $99 \%$ of its initial performance in $85 \%$ relative humidity at room temperature for $1000 \mathrm{~h}$ (with encapsulation) and at a thermal-stress temperature of $70{ }^{\circ} \mathrm{C}$ for $100 \mathrm{~h}$ (without encapsulation), respectively, outperforming BA-based PSCs [36]. Li et al. further explored the stability of unencapsulated (PDA)(MA) ${ }_{3} \mathrm{~Pb}_{4} \mathrm{I}_{13}$-based PSCs under various harsh environmental stresses, including ambient, heat and continuous illumination conditions. It was found that the weak van der Waals interactions between adjacent interlayers in RP phase perovskites could not adequately maintain the stability of layered structures. However, the stronger hydrogen bonding between alkyl diammonium ligands and inorganic layers in DJ phase perovskites means that these are more promising absorber materials for the fabrication of highly stable solar cells. For example, the unencapsulated PSCs containing (PDA)(MA) ${ }_{3} \mathrm{~Pb}_{4} \mathrm{I}_{13}$ exhibited good stability, as they maintained more than $95 \%$ of its initial performance under stress exposure in ambient conditions for $4000 \mathrm{~h}$, at a relative humidity of $85 \%$ and at a temperature of $85^{\circ} \mathrm{C}$ for $168 \mathrm{~h}$, and under continuous light irradiation for $3000 \mathrm{~h}$ (Figure $3 \mathrm{H}$ ). In contrast, the stability of devices based on $3 \mathrm{D}$-phase $\mathrm{MAPbI}_{3}$ and RP phase $(\mathrm{PA})_{2}(\mathrm{MA})_{3} \mathrm{~Pb}_{4} \mathrm{I}_{13}$ (propylamine abbreviated as $\mathrm{PA}$ ) was not adequate[35].

Similar stability benefits of DJ phase perovskites were also reported by Huang and coworkers. Besides the elimination of unstable van der Waals gap between interlayers, the improved film crystallinity and suppressed trap states in BDAMA-based $(n=5)$ films also contributed to the improved device longevity. The BDA-based layered PSC retained $84 \%$ of its original efficiency after $1182 \mathrm{~h}$ of exposure to $60 \%$ relative humidity, while its BA-based and 3D counterparts retained only 73 and 39\% of their original efficiency after 1176 and $1008 \mathrm{~h}$ of exposure, respectively [34]. Furthermore, the ordered crystal orientation, enhanced crystallinity and improved charge transport in the BDA-based perovskite led to a substantially better PCE for the corresponding device $(17.91 \%)$ than that of the BA-based system (15.10\%). Tailoring the alkyl chain length of linear organic ligands was also proven to help regulate crystal distortion, crystal orientation and QW distributions in these DJ phase layered perovskites. Huang et al. further systematically explored the structural characteristics of DJ phase perovskites based on a series of linear diammonium cations with various chain lengths, such as PDA, BDA, 1,5-pentamethylenediamine (PeDA) and HDA [63]. It was found that in contrast to the PeDA- and HDA-based films, the DJ phase perovskites using PDA and BDA as spacer cations have shorter QW barrier thickness and thus less structural distortion and a more uniform QW distribution (Figure 3F). The authors attributed the serious phase diversity in PeDA and HDA films to the low solubility of these long-chain cations. Low $n$-value phases deposited first during the crystallization process, forming a thermodynamically active solubility barrier that suppressed the direct formation of higher $n$-value phases, resulting 
in a multiphase film comprising non-stoichiometric molar proportions of components. This preferred film morphology and crystalline orientation, together with the flattened energy landscape afforded by the bulky shorter chain-length ligands, led to the formation of continuous channels for efficient charge transport and extraction in the vertical direction. Thus, the DJ phase PDA- and BDA-based PSCs delivered higher PCEs of 14.16 and $16.38 \%$, respectively, outperforming the PeDA and HDA analogs, which had PCEs of less than $13 \%$.

Inspired by the success of fluorine substitution for improving the efficiency of organic solar cells [91], recent research has focused on the fluorination of organic spacers. You et al. selectively monofluorinated PEA at different positions, and demonstrated that 2D perovskites that used monofluorinated PEAs as spacer cations had different packing arrangements and oriented order, which related to their formation energies [92]. Through the chemical modification of spacer cations, the differences in packing motif of the organic layers with varying degrees of orientational disorder were obtained, which were resulted from the perturbations in noncovalent interactions between bulky organic cations. The more favorable formation energies of 2D phases would lead to improved crystal growth, resulting in regular phase distributions, improved morphologies and well-oriented crystals in these 2D perovskites, and corresponding devices with good performance. Similar results were reported by $\mathrm{Zhu}$ et al., who discovered that a 4-fluorophenylethylammonium (F-PEA)based organic layer exhibited shorter average ringcentroid-to-centroid distances and enhanced orbital interactions between the inorganic layers. This led to the formation of the preferred stacking alignment of organic layers and perovskite sheets, which resulted in good interlayer electronic coupling, out-of-plane conductivity and low trap density. Consequently, a PCE of $13.64 \%$ and good thermal stability were exhibited by (F-PEA) ${ }_{2} \mathrm{MA}_{4} \mathrm{~Pb}_{5} \mathrm{I}_{16}$ based devices[93]. However, such a fluorine substitution strategy has not been applied for the development of DJ phase 2D perovskites. Thus, the use of modern synthetic chemistry techniques to add functional halogen or sidechain substituents to organic ligands should allow access to a new class of 2D perovskites for elaboration into bandgaptunable photovoltaic devices, although such approaches are yet to be widely exploited in solar cell applications [54]. The future design of such functionalized organic cations and the determination of the intermolecular interactions associated with inner properties will play an essential role in the further development of 2D perovskite materials, and in achieving higher PCEs and stabilities in the corresponding devices.

\subsection{QW-thickness control}

Increasing the stacking-thickness number of symmetrical inorganic corner-sharing octahedra layers within 2D perovskites is an effective strategy for weakening the dielectric confinement effect and broadening their electronic bands to reduce quantum confinement in naturally formed QW structures. This decreases exciton binding energy and enables tuning of optoelectronic properties, endowing the corresponding devices with higher efficiencies. In general, the average $n$ value in 2D perovskites is defined by the stoichiometric ratios of different raw components in their precursor solutions, while in 3D perovskites, the value of $n$ is regarded as infinite. A direct relationship exists between the value of $n$ and the shortcircuit current density $\left(U_{s c}\right)$, and this has been studied by different groups [49, 94]. Modeling of simulated and experimental $J-V$ curves indicated that weak absorption and a wide bandgap are detrimental to the performance of perovskite devices containing lower $n$-value phases (i.e., $n \sim 1$ ). In devices containing higher $n$-value phases $(n \geq 6)$, the main limiting factor is reduced mobility, which leads to large radiative recombination loss. It was concluded from the observed performances of $(\mathrm{BA})_{2}(\mathrm{MA})_{n-1} \mathrm{~Pb}_{n} \mathrm{I}_{3 n+1}$ based devices that $J_{s c}$ increased with $n$, from $0.06 \mathrm{~mA} \mathrm{~cm}^{-2}$ (for $n=1$ ) to $9.09 \mathrm{~mA} \mathrm{~cm}^{-2}$ (for $n=4$ ) [14]. An analogous observation was made in a study of DJ phase PSCs that incorporated PDMA, in which $J_{s c}$ increased from 11.5 to $21.5 \mathrm{~mA} \mathrm{~cm}^{-2}$ as the value of $n$ increased from 3 to $10[78,79]$. Specifically, Etgar et al. reported that the DJ phase perovskite (PDMA) $\left(\mathrm{Cs}_{0.05} \mathrm{MA}_{0.15} \mathrm{FA}_{0.8}\right)_{9} \mathrm{~Pb}_{10}\left(\mathrm{I}_{0.93} \mathrm{Br}_{0.07}\right)_{31}$ with $n=10$ showed a comparable carrier mobility to its 3D counterpart [79]. It was also observed that increased $n$-values in PDMA-based DJ phase perovskites can result in changes of film color and a red shift of emission peaks in PL spectra (Figure 4A). The increased full width at half maximum (FWHM) of PL peaks for the $n \geq 2$ PDMA-based DJ phase perovskite films indicated that these comprised mixed phase compositions. Furthermore, increased QW thicknesses induced changes in band alignment. Huang et al. determined the energy levels of BDA- and PeDA-based DJ phase perovskites with $n=1-6$ by ultraviolet photoemission (UPS) spectroscopy. This revealed that the valence and conduction bandwidths deepened and the optical bandgap gradually decreased as $n$-values increased. The BDA-based DJ phase perovskite displayed a flattened energy landscape, whereas the PeDA-based case presented a ladder-like pattern of disordered energy alignment (Figure 4B and C) [63]. However, increase in the thickness of inorganic frameworks can damage the structural stability of crystals, which means that the value 

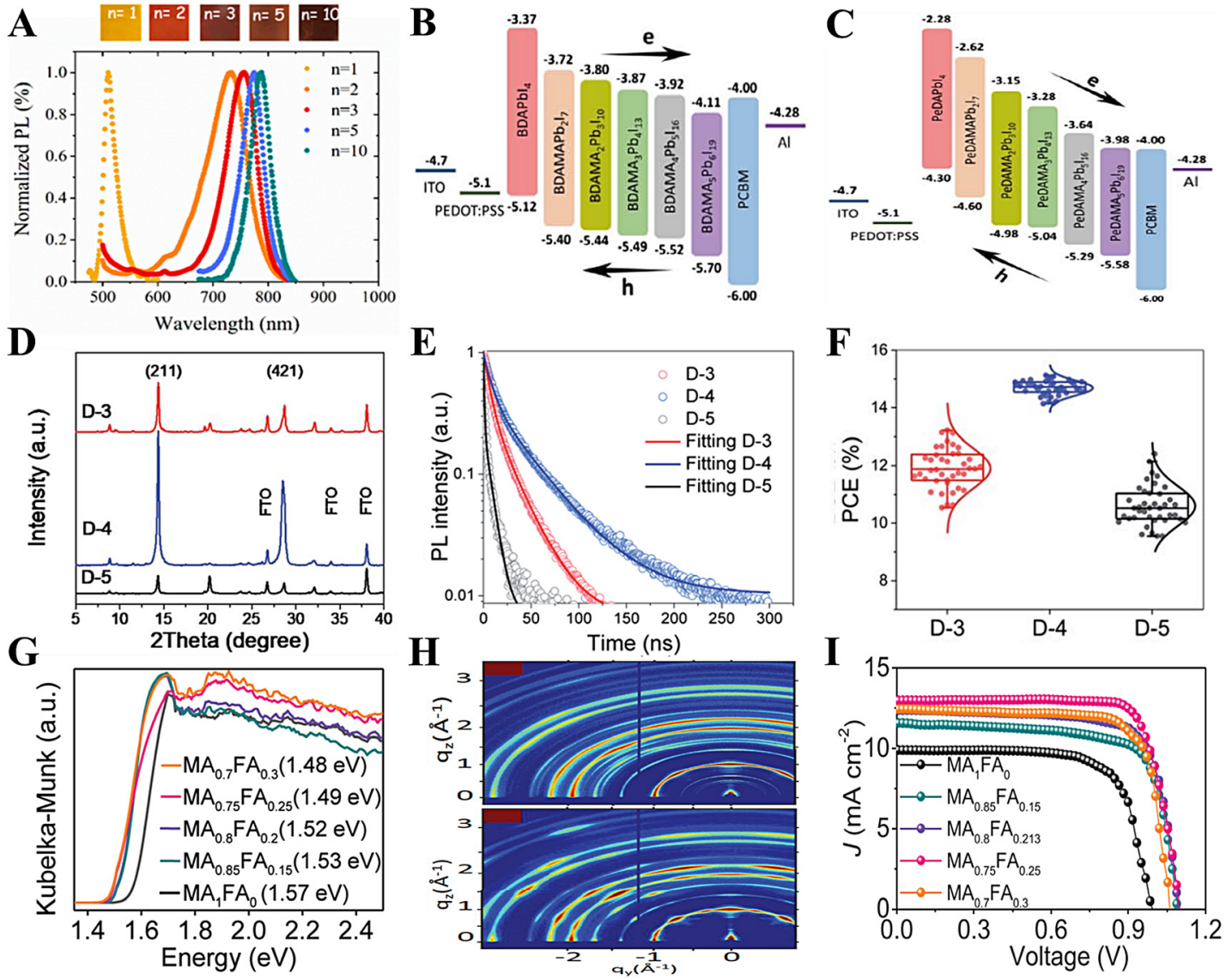

Figure 4: QW thickness control and mixed cation engineering in DJ phase perovskites.

(A) The increased $n$ values of PDMA-based DJ phase layered perovskites resulted in the changed film color and a red shift of emission peaks of PL spectra. Reproduced with permission [79]. Copyright 2019, American Chemical Society Publications. (B-C) Energy level diagrams of BDAand PeDA-based DJ phase perovskites with $n=1-6$. Reproduced with permission [63]. Copyright 2019, Wiley-VCH Publications. (D) XRD patterns of the (DMAPA)MA ${ }_{2} \mathrm{~Pb}_{3} \mathrm{I}_{10}$ (D-3), (DMAPA)MA $\mathrm{Pb}_{4} \mathrm{I}_{13}$ (D-4), and (DMAPA)MA ${ }_{4} \mathrm{~Pb}_{5} \mathrm{I}_{16}$ (D-5) thin films on the FTO substrate after annealing. (E) Time-resolved photoluminescence (TRPL) spectra of the D-3, D-4, and D-5 thin films. (F) PCE statistics of solar cells with a planar architecture of FTO/ $\mathrm{TiO}_{2}$ /perovskite/Spiro-OMeTAD/Au. Reproduced with permission [69]. Copyright 2020, Royal Society of Chemistry. (G) The absorption spectra of (3AMP) $\left(\mathrm{MA}_{1-x} \mathrm{FA}_{x}\right)_{3} \mathrm{~Pb}_{4} \mathrm{I}_{13}(x=0-0.3)$ crystals. (H) GIWAXS patterns of the (3AMP)(MA) ${ }_{3} \mathrm{~Pb}_{4} \mathrm{I}_{13}$ film (top) and (3AMP) $\left(M_{0.75} \mathrm{FA}_{0.25}\right)_{3} \mathrm{~Pb}_{4} \mathrm{I}_{13}$ film (bottom) deposited on FTO/PEDOT:PSS substrates. (I) $J-V$ curves of $(3 A M P)\left(M A_{1-x} \mathrm{FA}_{x}\right)_{3} \mathrm{~Pb}_{4} \mathrm{l}_{13}(x=0-0.3)$ solar cells. Reproduced with permission [65]. Copyright 2019, Wiley-VCH Publications.

of $n$ should be carefully considered to obtain a balance of stability and performance in devices.

Thus far, high-performance and stable layered PSCs have typically occupied $n$ values between 3 and 5, with 2D structures largely maintained with $n \leq 4$. Higher $n$-values resulted in layered perovskite films with features approaching those of the corresponding 3D phase; accordingly, such films are classified as quasi-2D phases [25]. Other research indicated that the number of inorganic slabs in single crystals with $n \leq 4$ was equal to the $n$-value of the precursor mixture, and that $n=5$ single crystals were only $90 \%$ purity [95]. The performances of devices based on layered perovskites with extremely high $n$-values were comparable to those of their 3D counterparts. For example, the efficiencies for the (PEA) $)_{2}(\mathrm{MA})_{n-1} \mathrm{~Pb}_{n} \mathrm{I}_{3 n+1}$-based perovskite, in which $n=60$, and the $\mathrm{BA}_{0.09}\left(\mathrm{FA}_{0.83} \mathrm{Cs}_{0.17}\right)_{0.91} \mathrm{~Pb}\left(\mathrm{I}_{0.6} \mathrm{Br}_{0.4}\right)$-based perovskite, in which were 15.2 and $17.2 \%$, respectively $[49,96]$. However, the overall thickness of inorganic slabs is beyond the limited thickness of conventional 3D nanocrystals [97]. It is therefore more correct to classify inorganic slabs as bulk heterostructures, or regard the spacer cations as dopants that passivate the slab trap states and enhance the longterm stability of devices. Although synergetic improvements in device performance have been obtained in layered perovskites with high $n$-values, the effect of QW-thickness on the phase distributions and crystal orientations of DJ phase 2D perovskites has not been systematically determined, and further detailed research is required. 
Solution-processed layered perovskites with higher $n$-values $(n \geq 3)$ generally suffer from phase diversity and random QW distributions, which may be related to variations in the thermodynamic stability and formation energy of 2D phases with different thicknesses [98]. Moreover, it has been observed that mixed-cation DJ phase perovskites with varied $n$-values could influence the purity of $\mathrm{FA}^{+}$ phase. That is, a series of optically inactive phases of FAand Cs-based perovskites were detected in lower $n$-value films using PDMA-based triple-cation layered perovskite, whereas the high $n$-value film exhibited good phase purity and stabilized the black phase of $\mathrm{FAPbI}_{3}$. As for the QW distribution in such PDMA-based layered perovskites, the increased crystal dimensions by elevating the $n$ values could shorten the diverse phase distribution of QWs. Interestingly, the authors used the ${ }^{1} \mathrm{H}$ nuclear magnetic resonance (H NMR) spectroscopy to accurately determine the ratio of the small and bulk organic cations, which provided a useful method to obtain the detailed component information compared with the initial stoichiometric ratio in the precursor solution. By scratching the perovskite powder from the already deposited films and integrating the peak of the protons, the $n=10$ perovskite films exhibited a ca. 1:10 ratio of PDMA and FA, which corresponded to the component of $n=11$ [79]. The changed $n$ values from precursor solution to final deposited film would also take an influence on the film quality. Yuan et al. found that DJ phase (PXD)(MA) ${ }_{n-1} \mathrm{~Pb}_{n} \mathrm{I}_{3 n+1}$ (p-xylylenediamine abbreviated as PXD) films had extensive acicular morphology when $n=4$ or 5 , whereas those films with lower $n$-values had more compact surface coverage [71]. A similar effect of $n$-values on stoichiometric ratios and thus morphology was also observed in DJ phase (DMAPA)MA ${ }_{n-1}$ $\mathrm{Pb}_{n} \mathrm{I}_{3 n+1}$ (3-(dimethylammonium)-1-propylammonium abbreviated as DMAPA) perovskites. The $n=3$ film exhibited heterogeneous grain sizes throughout, but the $n=4$ film exhibited a uniform grain distribution with a vertical arrangement. Further increased the $n$-value led to the appearance of large pinholes on the surface and reduced film crystallinity (Figure 4D). The authors attributed the morphology and crystallinity improvements in these DMAPA-based perovskites to the higher $n$-value precursors exhibiting enhanced intermediate-related crystallization kinetics during the hot-casting process. Specifically, it was proposed that an optimal concentration of spacer cations would result in rapid formation of the intermediate phase, and this phase would serve as a high-quality template to guide the subsequently homogeneous crystallization of layered perovskites. Deviation from this optimal concentration would perturb the formation and decomposition of intermediate phases, leading to layered perovskites with the deteriorated phase distributions, crystal orientations and charge transport properties [69]. The $n=4$ films therefore exhibited a lower trap density and a longer charge lifetime with suppressed non-radiative recombination, in contrast to the $n=3$ and $n=5$ films (Figure 4E). In terms of device efficiencies, the PSCs based on $n=4$ films had better reproducibility and a high average efficiency of $14.68 \%$, whereas the PSCs based on $n=3$ and $n=5$ films delivered an average efficiency of 11.91 and 10.60\%, respectively (Figure 4F).

\subsection{Multi-cation engineering}

Multi-cation engineering of 3D perovskites has been widely explored and used to fabricate efficient and stable PSCs $[99,100]$. The design of new types of 2D perovskite materials with mixed cations has drawn on this engineering knowledge to improve the optoelectronic properties and efficiency of charge transport in QW structures, and thus further enhance the performance of 2D PSCs. For example, the grain size and surface quality of $(\mathrm{BA})_{2}(\mathrm{MA})_{3} \mathrm{~Pb}_{4} \mathrm{I}_{13}$ films were improved by partial replacement of $\mathrm{MA}^{+}$with $\mathrm{Cs}^{+}$, and a perfect crystalline orientation was obtained. A growth kinetics study revealed that the $\mathrm{Cs}^{+}$doping slowed the crystallization kinetics during solution-casting, resulting in improved optoelectronic properties in the corresponding devices: e.g., a PCE improvement from 12.3 to $13.7 \%$ was achieved upon 5\% $\mathrm{Cs}^{+}$doping [27]. In similar work, Zhou et al. incorporated $\mathrm{FA}^{+}$into a $(\mathrm{BA})_{2}(\mathrm{MA})_{3} \mathrm{~Pb}_{4} \mathrm{I}_{13}$ film and explored the influences of this organic cation substitution on the morphology, crystal orientation and crystallization kinetics of the resulted RP phase 2D perovskite. It was found that an FA-doping level of $20 \%$ optimized the carrier lifetime and crystal orientation, and the corresponding device delivered a PCE of $12.8 \%$ [101].

Recently, such a strategy has been further developed to fabricate DJ phase 2D perovskites. Kanatzidis et al. used a mixed A-site cation strategy to tune the optical and electronic film properties of a DJ phase (3AMP)(MA) ${ }_{3} \mathrm{~Pb}_{4} \mathrm{I}_{13}$ perovskite, which improved the corresponding device performance. It was then found that the introduction of $\mathrm{FA}^{+}$, which is larger than $\mathrm{MA}^{+}$, gave a (3AMP) $\left(\mathrm{MA}_{0.75^{-}}\right.$ $\left.\mathrm{FA}_{0.25}\right)_{3} \mathrm{~Pb}_{4} \mathrm{I}_{13}$ film featured on the larger $\mathrm{Pb}-\mathrm{I}-\mathrm{Pb}$ angles in its crystal structure and a narrower optical bandgap than the (3AMP)(MA) ${ }_{3} \mathrm{~Pb}_{4} \mathrm{I}_{13}$ film, which enabled broader absorption in the visible light region (Figure 4G) [65]. The optimal vertical crystal orientation against the substrate was also observed in the (3AMP) $\left(\mathrm{MA}_{0.75} \mathrm{FA}_{0.25}\right)_{3} \mathrm{~Pb}_{4} \mathrm{I}_{13}$ film, whereas the (3AMP)(MA) $)_{3} \mathrm{~Pb}_{4} \mathrm{I}_{13}$ film exhibited distinct diffraction rings in its GIWAXS pattern and therefore had a more random crystal orientation (Figure 4H). Under the optimal incorporating amount of FA cation, the PCE of the 
(3AMP) $\left(\mathrm{MA}_{0.75} \mathrm{FA}_{0.25}\right)_{3} \mathrm{~Pb}_{13}$ device reached to $11.24 \%$, which was better than that of the (3AMP)(MA) ${ }_{3} \mathrm{~Pb}_{4} \mathrm{I}_{13}$-based device with a PCE of $7.02 \%$ (Figure 4I). Moreover, the presence of $\mathrm{FA}^{+}$increased the overall stability of the resulting films and devices under humidity and illumination conditions, surpassing the stabilities of the RP phase 2D perovskite and their 3D analogs. Chen et al. further improved the efficiency of (3AMP) $\left(\mathrm{MA}_{0.75} \mathrm{FA}_{0.25}\right)_{3} \mathrm{~Pb}_{4} \mathrm{I}_{13}$ based PSCs to $16.25 \%$ with the aid of an MA-thiocyanate (SCN) additive [66]. In addition, Loo et al. replaced the A-site $\mathrm{MA}^{+}$with a mixed alloy, and achieved a remarkable PSC efficiency of $18.2 \%$ for the (BDA) $\left(\mathrm{Cs}_{0.1} \mathrm{FA}_{0.9}\right)_{4} \mathrm{~Pb}_{5} \mathrm{I}_{16}$ based device [75].

For the relatively large monoammonium cation, the guanidinium $\mathrm{GA}^{+}\left(\mathrm{C}\left(\mathrm{NH}_{2}\right)_{3}{ }^{+}\right)$has also been used to regulate the structural properties of $2 \mathrm{D}$ perovskite systems. $\mathrm{Xu}$ et al. found that $\mathrm{GA}^{+}$could occupy the $\mathrm{BA}^{+}$position in a $\mathrm{BA}_{2} \mathrm{MA}_{2} \mathrm{~Pb}_{3} \mathrm{I}_{10}$ perovskite, and this enhanced the out-ofplane orientation of QWs. The presence of $\mathrm{GA}^{+}$also relaxed the lattice strain relaxation, resulting in the inhibition of phase segregation and decomposition under an ambient atmosphere, and a much improved operational stability [102]. Triple-cation CsMAFA-based 3D perovskites are regarded as one of the most promising candidates for high-performance photovoltaic applications. Zhu et al. found that triple-cation doping led to better 2D perovskite-based device performance than mono- or binary-cation doping. Thus, a mixed perovskite system assembled by doping $\mathrm{MA}^{+}, \mathrm{FA}^{+}$and $\mathrm{Cs}^{+}$into $(\mathrm{BA})_{2}(\mathrm{MA})_{4} \mathrm{~Pb}_{5} \mathrm{I}_{16}$ exhibited improved morphology and crystallinity relative to its non-doped precursor, resulting in enhanced optoelectronic properties and a high PCE $(14.23 \%)$ in the corresponding device [103]. Etgar et al. synergistically tailored A-site cations and $\mathrm{X}$-site anions to achieve a PCE of $15.6 \%$ for (PDMA) $\left(\mathrm{Cs}_{0.05} \mathrm{MA}_{0.15^{-}}\right.$ $\left.\mathrm{FA}_{0.8}\right)_{9} \mathrm{~Pb}_{10}\left(\mathrm{I}_{0.93} \mathrm{Br}_{0.07}\right)_{31}$-based DJ phase PSCs, which also had carrier mobility comparable to their 3D counterparts [79]. A similar doping strategy has also been applied to spacer cations. Zhou et al. used a mixture of two spacer cations, namely 2,2,2-trifluoroethylamine $\left(\mathrm{F}_{3} \mathrm{EA}^{+}\right)$and $\mathrm{BA}^{+}$, to form a low-dimensional perovskite. The electronegativity of fluorine in the $\mathrm{F}_{3} \mathrm{EA}^{+}$cation led to a high dipole moment, which inhibited charge recombination and increased the dissociation of electron-hole pairs. This increased the open-circuit voltage and fill factor, and the $\left[(\mathrm{BA})_{0.94}\left(\mathrm{~F}_{3} \mathrm{EA}\right)_{0.06}\right)_{2}(\mathrm{MA}]_{3} \mathrm{~Pb}_{4} \mathrm{I}_{13}$-based PSC had an outstanding PCE of $12.51 \%$ [104]. Chen et al. introduced $\mathrm{PEA}^{+}$as a second spacer cation, which induced aggregation in the precursor solution, leading to a 2D $(\mathrm{BA})_{2}(\mathrm{MA})_{4} \mathrm{~Pb}_{5} \mathrm{I}_{16}$ perovskite system that had good crystal orientation and a large grain size $(>1 \mu \mathrm{m})$ [105]. However, much is unknown on the effects of second spacer-cation doping in DJ phase perovskites.

As can be seen, the multi-cation engineering of DJ phase perovskites enables control of their preferred out-ofplane orientation, which optimizes crystal quality and enhances the long-term stability of the corresponding devices. Further study is needed to identify the mechanistic effects of variations in the types and proportions of cations in mixed $\mathrm{A}^{\prime}$ - and/or A-site groups on crystallization kinetics and film properties, such as crystal morphology, quality, dimensions and orientation, and on corresponding device performance. This knowledge will help to broaden the categories of new 2D perovskite materials, and enable the realization of high-performance 2D perovskite optoelectronic devices.

\subsection{Additive engineering}

The inferior device performance of $\mathrm{DJ}$ phase 2D perovskites compared to that of their 3D counterparts, particularly with regard to $J_{s c}$ values. This mainly originates from the undesirable charge transport efficiency, which is attributable to the large exciton binding energy in DJ phase perovskites and the inhomogeneity of film morphology and randomness of crystal orientation. The irregular QW alignment and large energy barrier also limit charge transport and separation, resulting in severe charge accumulation and recombination loss. Chemical doping of 2D perovskite precursors has been reported to be an effective approach to overcome these limitations and provide additional optoelectronic properties and device stability.

Chen and coworkers reported a new type of full FA-based DJ phase perovskite with the general formula (PDA)(FA) ${ }_{n-1} \mathrm{~Pb}_{n} \mathrm{I}_{3 n+1}$ [70]. $\mathrm{FACl}$ was used as an additive during film preparation, which prevented the formation of $\delta$-phase $\mathrm{FAPbI}_{3}$ and $\mathrm{PbI}_{2}$ byproducts in the annealing process. GIWAXS was used to examine the role of $\mathrm{Cl}^{-}$in the conversion of the disordered precursor colloidal gel to an annealed film, and showed that the Cl-containing intermediates and $\delta$-phase $\mathrm{FAPbI}_{3}$ appeared first in the initial precursor state. Then, under continuous thermal annealing, rapid ( $\leq 10 \mathrm{~s}$ ) phase conversion from the $\delta$-phase to the $\alpha$-phase occurred, accompanied by transformation of the Cl-containing intermediates to a $2 \mathrm{D}$ perovskite phase via $\mathrm{I}^{-} / \mathrm{Cl}^{-}$ion exchange (Figure $5 \mathrm{~A}$ and $\mathrm{B}$ ). This $\mathrm{FACl}$ addition led to (PDA)(FA) ${ }_{3} \mathrm{~Pb}_{4} \mathrm{I}_{13}$ thin films with vertical-oriented crystal grains, compact morphologies, well-matched energy-bandgap level alignments and enhanced optoelectronic properties. Ultimately, the (PDA)(FA) ${ }_{3} \mathrm{~Pb}_{4} \mathrm{I}_{13}$ based PSC achieved a promising PCE of $13.8 \%$ and had good 
A
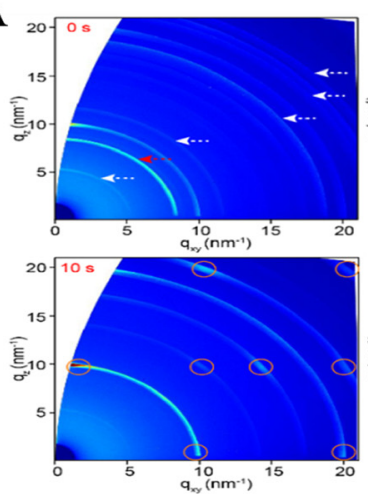

C

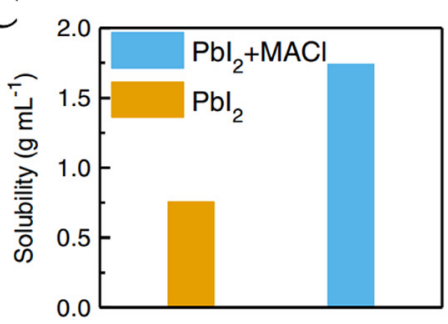

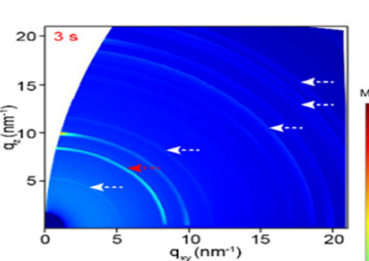

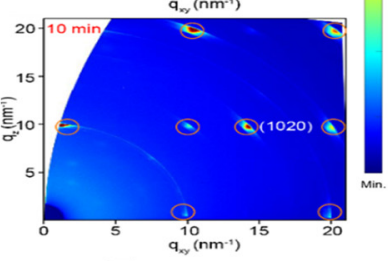

D

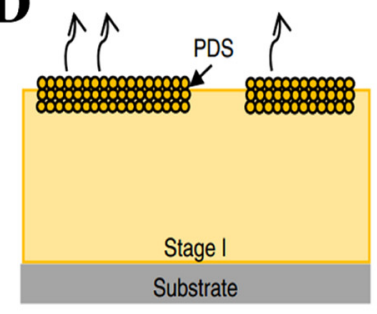

B
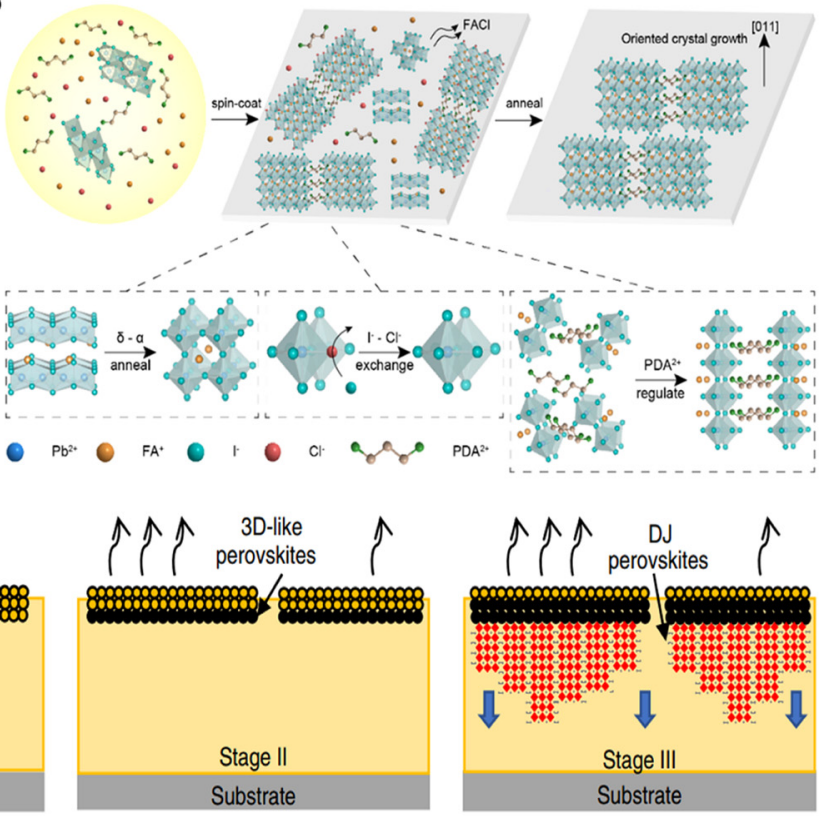

Figure 5: Additive engineering.

(A) The $2 \mathrm{D} \mathrm{GI}-\mathrm{XRD}$ patterns of the (PDA)(FA) ${ }_{3} \mathrm{~Pb}_{4} \mathrm{l}_{13}$ perovskite films annealed at $150{ }^{\circ} \mathrm{C}$ for $0,3,10 \mathrm{~s}$ and $10 \mathrm{~min}$, respectively. The red dotted arrows were belong to the $\delta$-phase $\mathrm{FAPbl}_{3}$, the white dotted arrows were belong to the $\mathrm{Cl}$-containing intermediates and the orange circles showed the Bragg peak positions of the (PDA)( $\mathrm{FA})_{3} \mathrm{~Pb}_{4} \mathrm{l}_{13}$ perovskite phase. (B) Schematic illustration illustrating the microscopic growth mechanism of the DJ phase 2D perovskite film under the assistance of $\mathrm{FACl}$ additive. Reproduced with permission [70]. Copyright 2019, WileyVCH Publications. (C) The solubility of $\mathrm{Pbl}_{2}$ in DMF and $\mathrm{Pbl}_{2}$ with $0.5 \mathrm{MACl}$ in DMF. (D) Schematics of the precipitation of DMF-based solvated phase (PDS) at the liquid surface, which triggered the formation of 3D-like perovskites on soaked PDS surface and then induced the directional growth of DJ phase layered perovskites. Reproduced with permission [71]. Copyright 2020, Wiley-VCH Publications.

thermal stability, as ca. $85 \%$ of its original PCE remained after $1000 \mathrm{~h}$ under $85^{\circ} \mathrm{C}$ thermal stress.

Alex et al. found that addition of $\mathrm{NH}_{4} \mathrm{SCN}$ to the DJ phase quasi-2D (BDA)(MA) ${ }_{4} \mathrm{~Pb}_{5} \mathrm{I}_{16}$ perovskite facilitated vertical crystal growth on a substrate [74]. Moreover, the QW distribution in the $\mathrm{SCN}^{-}$-modified perovskites was narrowed and concentrated into thermodynamically favorable $n=3$ and $n=4$ phases. This improved the crystal orientation and enhanced the phase purity, leading to fast charge transport and a high PCE (14.53\%) in the corresponding DJ phase PSCs. It was suggested that the lone pair of electrons on the $S$ and $\mathrm{N}$ atoms in $\mathrm{SCN}^{-}$would act as Lewis bases and thus interact with the Lewis acid $\mathrm{Pb}^{2+}$ and bond to $\left[\mathrm{PbI}_{6}\right]^{4-}$. This would result in the formation of negatively charged $\mathrm{SCN}^{-}$containing perovskite colloids, which could repel each other in the precursor solution, facilitating dimensional control and a narrower phase distribution. In addition, it was suggested that the interaction between charged substrates and precursor/colloids may induce the vertical orientation of the layered perovskites. A further observation was that the surface charge of the $\mathrm{SCN}^{-}$-modified perovskite film changed from positive (on glass) to neutral (on poly [bis(4-phenyl)(2,4,6-trimethylphenyl)amine]) and negative (on PEDOT:PSS, and on PTAA with 2,3,5,6-tetrafluoro7,7,8,8-tetracyanoquinodimethane, F4TCNQ), with the crystal orientation perpendicular to the substrate and the parallel QW arrangement diminished in the latter two filmsubstrate combinations. This indicates that electrostatic interactions between substrates and additives represent a new avenue to explore to evaluating the relationship between interfacial properties and the characteristics of additives and crystal orientations, which will assist in the rational choice of additives to form desirable DJ phase 2D PSCs.

Wu and coworkers used an MASCN additive to fabricate a high-quality DJ phase (3AMP) $\left(\mathrm{MA}_{0.75} \mathrm{FA}_{0.25}\right)_{3} \mathrm{~Pb}_{4} \mathrm{I}_{13}$ perovskite film [66]. The incorporation of an optimal proportion of MASCN induced the preferred crystalline orientation along the (011) plane, perpendicular to the substrate. In addition, the optimized DJ phase 2D perovskite film exhibited high crystallinity, few trap states and improved band alignment, which enabled efficient charge transfer from the absorber to the charge transport layer. In contrast, the film without MASCN modification comprised randomly stacked crystal grains. The MASCN-modified (3AMP) $\left(\mathrm{MA}_{0.75} \mathrm{FA}_{0.25}\right)_{3} \mathrm{~Pb}_{4} \mathrm{I}_{13}$ 
PSCs delivered a champion PCE of $16.25 \%$, and retained $80 \%$ of this PCE after 35 days of ambient storage. Although the positive effects were observed through additive incorporation, the excessive amount would deteriorate the film quality, leading to the rough and irregular surface morphology with pinholes and terrible interface contact.

As the above discussion elucidates, the manipulation of QW orientation and film crystallinity are key to achieving high-performance DJ phase 2D PSCs. However, the limited knowledge of the dynamics of crystallization during film formation may hinder effective regulation of $\mathrm{QW}$ growth and result in a gradient of QW thickness. To explore ways to prevent this, Yuan et al. systematically studied the effect of $\mathrm{MACl}$ as an additive on the crystal growth process of DJ phase perovskites, as $\mathrm{MACl}$ is compatible with various organic cation spacers [71]. It was found that $\mathrm{MACl}$ suppressed the precipitation of a $\mathrm{PbI}_{2}-$ DMF-based solvated phase (dominated by a $(\mathrm{MA})_{2}(\mathrm{DMF})_{2} \mathrm{~Pb}_{3} \mathrm{I}_{8}$ intermediate phase) in the precursor solution, with this phase instead concentrating on the solution surface (stage I), due to the increased solubility of $\mathrm{PbI}_{2}$ in DMF containing MACl (Figure 5C). 3D-like perovskites subsequently formed at the liquid-air interface (stage II), where they served as templates and induced the direct downward growth of DJ phase perovskites with preferred out-of-plane orientations (stage III) (Figure 5D). However, without the addition of $\mathrm{MACl}$, there was excessive precipitation of the solvated phase in the bulk precursor, resulting in a randomly oriented growth template and a heterogeneous $\mathrm{QW}$ arrangement. The precipitation of the solvated phase also influenced the final QW distribution. The addition of $\mathrm{MACl}$ regulated the phase purity by increasing the proportion of low $n$-value $(n=2,3)$ phases and hindering the formation of high $n$-value phases. Similar phenomena were observed in ACI perovskites that contained $\mathrm{MACl}$, which generated a good gradientthickness distribution of QWs and therefore led to better energy alignment for charge transport [48]. As a result, a combination of ligand modification and additive engineering afforded the DJ phase (PXD)(MA) ${ }_{2} \mathrm{~Pb}_{3} \mathrm{I}_{10}$ PSCs with an efficiency of $15.6 \%$. Moreover, Kanatzidis et al. introduced $\mathrm{HI}$ into a precursor solution to improve the morphology and crystallinity of DJ phase perovskite films. HI disrupted the strong hydrogen bonding between solvents and spacer cations and thus modified the rates of solvent evaporation and perovskite crystallization [88].

In general, to be useful as an additive for 2D perovskites, a compound must ensure the enhanced surface morphology and crystallinity of final perovskite films, and generate highly connected crystal stacking with a perpendicular crystal orientation against the substrate. It is also imperative that additive-modified 2D perovskites exhibit better phase purity and more regular thickness alignment of QWs, such that they have more efficient charge extraction and transport, and lower potential barriers than their unmodified component, leading to better device performance. Although numerous additives have been applied to regulate crystallization kinetics and achieve preferred orientations in 2D perovskites, there is still lacks of a basic concept to guide the selection of such functional additives. The current group of additives used in DJ phase 2D perovskites is largely an extension of the group used to modify RP phase perovskites before, and mainly includes ammonium salts containing $\mathrm{Cl}^{-}(\mathrm{MACl}, \mathrm{FACl}$ and $\mathrm{NH}_{4} \mathrm{Cl}$ ) or SCN${ }^{-}$(MASCN and $\mathrm{NH}_{4} \mathrm{SCN}$ ). The compatibility of certain additives remains under investigation, and some may not be applicable with certain spacer cations or perovskite components. A continuous optimization process needs to attempt first before receiving an optimal recipe. Therefore, the further research around influencing mechanisms of additive molecular structure-film qualityQW arrangement-device performance is in urgent need to realize the controllable preparation of high-performance DJ phase PSCs.

\subsection{Solvent engineering}

Research on solvent engineering is ongoing, to identify methods that optimize the crystallization process of $2 \mathrm{D}$ perovskites and enable control of crystal growth in the outof-plane orientation. In general, the arrangement of spacer layers in 2D perovskite films is preferentially parallel to the substrate through the self-assembly formation process. This lack of out-of-plane orientation is believed to be a primary reason for the suppressed charge transport and limited performance of 2D PSCs compared to their 3D counterparts. In 3D perovskites, solvent variation is an effective way to control the crystallinity and morphology of films. For example, strong coordination between a polar aprotic solvent (a Lewis acid) and a metal halide (a Lewis base) can result in the formation of an intermediate phase adduct, thereby retarding the rate of crystallization and resulting in optimized film quality [106, 107]. A similar process regulation in the formation of $2 \mathrm{D}$ perovskites, with the use of certain solvents in precursor solutions leading to improvements in film quality and crystal orientation. Kanatzidis et al. were the first to describe a mixed solvent strategy combined with the hot-casting method. It was found that use of an appropriate amount of DMSO in a precursor solution led to better film crystallinity and crystal 
growth orientation in a $\mathrm{BA}_{2} \mathrm{MA}_{4} \mathrm{~Pb}_{5} \mathrm{I}_{16}(n=5)$ film. Solvent evaporation during the hot-casting process was accelerated by the formation of an intermediate solvated phase that rapidly escaped from the imperfect self-assembled portion. Thus, use of the optimized solvent system (3:1 DMF:DMSO) afforded a film with the desired microstructure, which exhibited excellent charge-extraction efficiency and yielded a device with an outstanding PCE (10\%) and less hysteresis than devices formed by processes using different solvent systems [25].

The effects of DMSO in 2D perovskite film were further explored by Wang et al., who found that DMSO-incorporating intermediate phases tended to form high $n$-value phases during the formation process, which reduced the polydispersity of phase distributions [108]. However, excess DMSO increased barriers to the formation of high $n$-value phases, and resulted in smaller differences in their thermodynamic stability, resulting in 2D perovskite films containing solvated phases and therefore of low phase purity [25]. In view of this, Zhao et al. demonstrated a template-induced nucleation process for the assembly of a $(\mathrm{BA})_{2}(\mathrm{MA})_{3} \mathrm{~Pb}_{4} \mathrm{I}_{13}$ perovskite, which was based on controlling the substrate temperature before the spin-casting process. This resulted in the RP phase $2 \mathrm{D}(\mathrm{BA})_{2}(\mathrm{MA})_{3} \mathrm{~Pb}_{4} \mathrm{I}_{13}$ perovskite rapidly and directly crystallizing from a disordered precursor solvate, without any intermediate-phase formation. The optimal substrate temperature and a
DMSO:DMF ratio of 7:3 yielded a film with a strongly preferential out-of-plane crystal orientation, a high phase purity and a large grain size. The resulting efficient carrier transport and long carrier lifetime led to the corresponding device exhibiting a good $J_{s c}$ and a PCE of $12.17 \%$. In contrast, indirect crystallization from the solvated phase led to a film with a broad phase distribution, resulting in corresponding devices with poor PCEs (3-8.5\%) [26].

Similar solvent engineering approaches have also been applied in the synthesis of DJ phase perovskites. Kanatzidis et al. used a DMF/DMSO solvent mixture and an $\mathrm{HI}$ additive in a precursor solution to fabricate high-quality (3AMP) $\left(\mathrm{MA}_{0.75} \mathrm{FA}_{0.25}\right)_{3} \mathrm{~Pb}_{4} \mathrm{I}_{13}$ films [65]. The DMSO helped to regulate the film morphology to ensure uniform and pinhole-free surface coverage, and also enhanced the optical absorption without influencing the bandgap. The synergistic effects of the mixed solvent and $\mathrm{HI}$ addition afforded a 2D perovskite film with optimized crystallinity and a vertical orientation with respect to the substrate (Figure 6A). The corresponding device achieved a much increased PCE of $11.19 \%$ than that of devices $(0.32 \%)$ obtained by a process with pure DMF as solvent (Figure 6B). DMSO-assisted film growth was also reported by Liu et al., who discovered that the strong coordination between DMSO and $\mathrm{PbI}_{2}$ in a precursor solution retarded the crystallization process and led to improvements in grain size, surface morphology and crystalline orientation in the
A
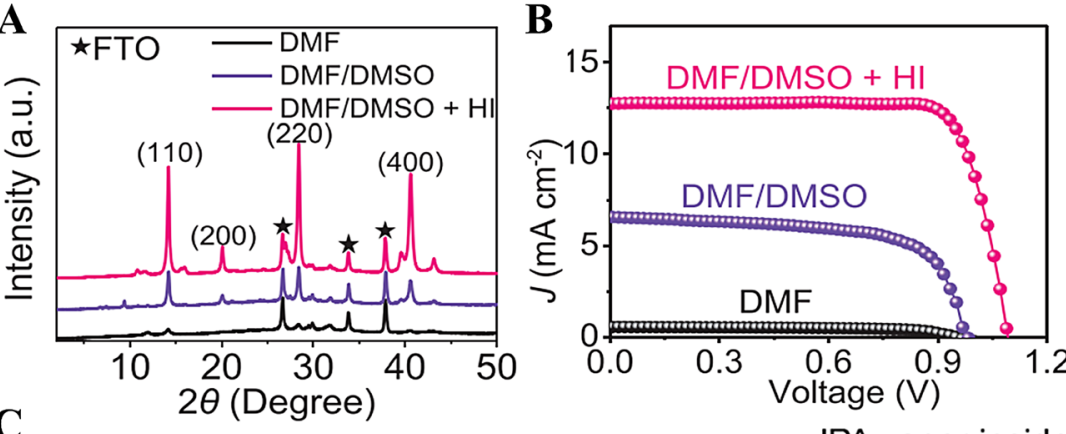

C

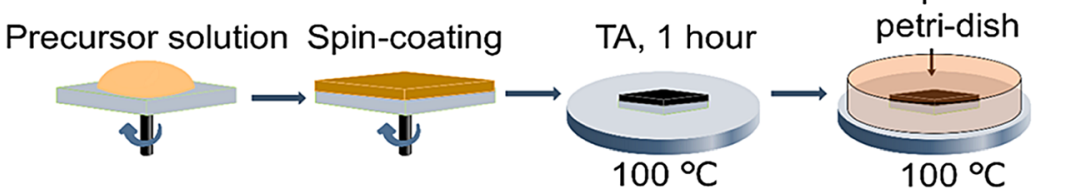

D Solvent vapor plasticizes the film. Vertical orientation at film surface templates structural re-arrangement Whole film develops towards thermodynamically-preferred orientation (vertical orientation)

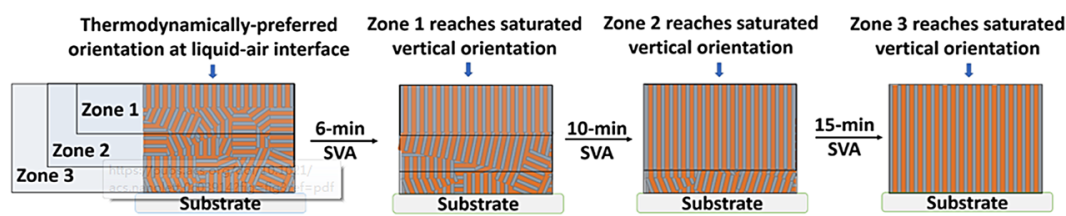

Figure 6: Solvent engineering.

(A) XRD patterns of (3AMP) $\left(\mathrm{MA}_{0.75} \mathrm{FA}_{0.25}\right)_{3} \mathrm{~Pb}_{4} \mathrm{I}_{13}$ perovskite films processed with different solvents. (B) J-V curves of the (3AMP) $\left(\mathrm{MA}_{0.75} \mathrm{FA}_{0.25}\right)_{3} \mathrm{~Pb}_{4} \mathrm{I}_{13}$ based solar cells using different solvents. Reproduced with permission [65]. Copyright 2019, Wiley-VCH Publications. (C) Scheme of isopropanol (IPA) solvent-vapor annealing (SVA) postdeposition processing of 2D perovskite films. (D) Illustration of the solvent-vapor plasticization process and the structural re-arrangement for vertical orientation during IPA post-treatment. Reproduced with permission [75]. Copyright 2020, American Chemical Society Publications. 
resulting DJ phase 2D perovskite [68]. Under a proper incorporation amount, DMSO could regulate the crystal growth, whereas the deteriorated film morphology was detected when the adding concentration was above the critical value. In a slightly different approach, Chen et al. used the low-polarity solvent dimethylacetamide (DMAC), due to its appropriate boiling point, to replace DMF and DMSO. It was discovered that the weak coordination of DMAC to the lead halide and ammonium salts effectively regulated the crystallization process, affording the $\mathrm{BA}_{2} \mathrm{MA}_{3} \mathrm{~Pb}_{4} \mathrm{I}_{13}$ perovskite with favorable crystal orientation and film quality, and the corresponding PSC exhibited a high PCE of $12.15 \%$ [109].

However, it is difficult to accurately control the substrate temperature in the hot-casting process, and this leads to poor reproducibility in the fabrication of devices, and means that this process may hard be used for large-scale fabrication. Hence, to meet the demands for better fabrication methods, there is an urgent need for a facile deposition procedure that does not require additives and high-temperature processing. Until this is achieved, solvent polarities and boiling points, and the interaction of components in immediate phases, must be carefully regulated to control the crystallization kinetics of 2D perovskites and thus obtain films with the preferred crystal orientation and crystallinity. In an alternative approach, Huang and coworkers developed the ionic liquid $n$-butylamine acetate (BAAc) to replace the traditional spacer cation BAI, and achieved a phase-pure preparation of QWs with a single well width. The strong coordination between BAAc and the inorganic framework in the precursor solution and the high viscosity and negligible vapor pressure of BAAc led to a regular, gelled distribution of intermediate compounds, which induced the uniform assembly of the QW segments [28].

Instead of using certain solvent ratios or solution components to access desired 2D perovskite materials from varied organic ligands and precursor compositions, Loo et al. reported a general solution-vapor annealing method that yields a broad range of vertically oriented $2 \mathrm{D}$ perovskites (Figure 6C). The authors used isopropanol (IPA) as a process solvent, as its vapor allowed the plasticization of perovskite films, and their structural reorganization and reorientation, without adversely affecting film quality [75]. Notably, (PEA) $)_{2}(\mathrm{MA})_{4} \mathrm{~Pb}_{5} \mathrm{I}_{16}$ film deposited from pure DMF had good phase purity without local compositional variation, and its PL spectra exhibited identical emission intensities and peak positions under both front-side and back-side excitation. In contrast, $(\mathrm{PEA})_{2}(\mathrm{MA})_{4} \mathrm{~Pb}_{5} \mathrm{I}_{16}$ film deposited from 4:1 DMF:DMSO had gradient-phase heterogeneity along its depth and a broad PL emission. The IPA post-treatment process maintained the uniform phase distribution of pure-DMF derived film, and also enhanced the out-of-plane orientation of crystals and therefore increased the electrical activity of the film. The surface of the pristine layered perovskite film also exhibited a more preferentially vertical orientation than the bulk, and this preference gradually deteriorated with depth. IPA vapor is sufficiently mobile to progressively plasticize the film from the surface to the bulk, and thermodynamically regulates the crystal reorientation and film quality with extended annealing time (Figure 6D). As a result, the IPA-processed $(\mathrm{PEA})_{2}(\mathrm{MA})_{4} \mathrm{~Pb}_{5} \mathrm{I}_{16}$ perovskite film exhibited better trap density and out-of-plane mobility than non-IPA-processed film. The good compatibility of this strategy was also exploited in the fabrication of DJ phase PSCs, outstanding PCEs of 16.5 and $18.2 \%$ were obtained from IPA-processed $(\mathrm{BDA})(\mathrm{MA})_{4} \mathrm{~Pb}_{5} \mathrm{I}_{16}$ - and $(\mathrm{BDA})\left(\mathrm{Cs}_{0.1} \mathrm{FA}_{0.9}\right)_{4} \mathrm{~Pb}_{5} \mathrm{I}_{16}$-based devices, respectively, which also showed superior environmental and operational stability to devices derived from non-IPA processed films.

\section{6 $\mathrm{Pb}$-free perovskite}

The development of $\mathrm{Pb}$-free perovskites, especially those in which $\mathrm{Pb}$ is replaced by $\mathrm{Sn}$, is regarded as a promising route for the environmentally friendly application and further commercialization of perovskite photovoltaic technology. However, Sn-containing perovskites suffer from p-type self-doping due to the ease with which $\mathrm{Sn}^{2+}$ is oxidized to $\mathrm{Sn}^{4+}$, which leads to nonuniform film morphology and series current leakage, and thus decreased device performance [110, 111].

Padture et al. were the first to use a Sn-containing DJ phase perovskite in a photovoltaic application, and the corresponding (4AMP)(FA) ${ }_{3} \mathrm{Sn}_{4} \mathrm{I}_{13}$ PSCs delivered a PCE of $4.22 \%$ [67]. Unencapsulated PSCs exhibited improved device stability, as they retained $89 \%$ of their original efficiency after $200 \mathrm{~h}$ under ca. $40 \%$ humidity atmosphere. Song et al. incorporated $\mathrm{BDA}^{+}$into the $\mathrm{FASnI}_{3}$ perovskite to regulate the crystallization process and suppress the oxidation of $\mathrm{Sn}^{2+}$ [76]. It was observed that $\mathrm{NH}_{3}{ }^{+}$penetrated deeply (0.923 $\AA$ ) into (BDA)FA ${ }_{n-1} \mathrm{Sn}_{n} \mathrm{I}_{3 n+1}(n>1)$, which increased the distance between a terminal $\mathrm{NH}_{3}{ }^{+}$and its nearest $\mathrm{I}^{-}$in inorganic slabs, weakened the hydrogen bonding and electrostatic attraction in the alternating layers and resulted in large $\mathrm{Sn}-\mathrm{I}-\mathrm{Sn}$ angles. These increased bond angles led to a highly symmetric crystal configuration in (BDA)FA ${ }_{2} \mathrm{Sn}_{3} \mathrm{I}_{10}$ (Figure 7A), which exhibited a bandgap that was almost as narrow as that in the $3 \mathrm{D} \mathrm{FASnI}_{3}$ perovskite. Similar results were also observed in 3AMP-based DJ phase perovskites [22]. The improved film morphology and 
reduced $\mathrm{Sn}^{4+}$-defect density in the $(\mathrm{BDA}) \mathrm{FA}_{2} \mathrm{Sn}_{3} \mathrm{I}_{10}$ film resulted in fast charge transfer from low $n$-value phases to 3D-like phases, leading to devices with a PCE of $6.43 \%$ and good durability when exposed to humidity, heat and illumination. In contrast, the 3D $\mathrm{FASnI}_{3}$ device had an efficiency of $4.20 \%$ and poor stability (Figure $7 \mathrm{~B}$ ). For instance, unencapsulated (BDA)FA $\mathrm{Sn}_{3} \mathrm{I}_{10}$ devices stored in an ambient atmospheric with ca. $40 \%$ humidity retained $89 \%$ of their initial PCEs after $200 \mathrm{~h}$, whereas 3D FASnI ${ }_{3}$ devices were completely degraded after $72 \mathrm{~h}$.

An unusual trend was generally detected in mixed $\mathrm{Pb} /$ Sn-based perovskites: bandgaps were found to be functions of the $\mathrm{Pb} / \mathrm{Sn}$ ratio, with lower ratios leading to a narrower bandgap and a high probability of the corresponding device exhibiting good efficiency [113]. Moreover, Kanatzidis et al. added $3 \mathrm{AMP}^{2+}$ into a narrow-bandgap $\mathrm{MA}_{0.5} \mathrm{FA}_{0.5} \mathrm{~Pb}_{0.5} \mathrm{Sn}_{0.5} \mathrm{I}_{3}$ system, and generated efficient mixed $\mathrm{Pb} / \mathrm{Sn}$-based PSCs from the resulting DJ phase perovskite. Notably, these demonstrated the suppression of $\mathrm{Sn}^{2+}$ oxidation and a long carrier lifetime over $650 \mathrm{~ns}$ [114]. Under the optimal 3AMP-doping concentration (2.5 mol\%), devices with an excellent PCE of $20.09 \%$ and good illumination stability was obtained, which maintained $73.2 \%$ of the original PCE efficiency after $100 \mathrm{~h}$ of continual light soaking in ambient air conditions. Kanatzidis et al. also used the large ethylenediammonium cation to decorate a mixed $\mathrm{Pb} / \mathrm{Sn}$ perovskite, which resulted in perovskite films with reduced trap density and increased chemical stability, although the 2D perovskite structure was absent in the resulting hollow films [82].

Overall, it is evident that Sn-based low-dimensional DJ phase perovskites have a stability advantage due to the strong hydrophobicity of their long and bulky organic cations and the strong interlayer interactions between their inorganic frameworks. Furthermore, the weaker coupling between $\mathrm{Sn}$ and I, compared to that between $\mathrm{Pb}$ and I, suppresses the formation of $\mathrm{Sn}$ vacancies and thereby ensures film conductivity. The comparability of the bandgaps of Sn-based low-dimensional DJ phase perovskites with those of their 3D analogs means that the former are also suitable as light absorbers in the bottom cells of tandem devices. Moreover, compared with films formed from their RP phase analogs, Sn-based DJ phase perovskites films have a greater charge-diffusion length, as result of their shorter interlayer distances, improved crystal symmetries and reduced spacer-cation proportions [21]. However, Snbased 2D perovskites containing multiple $\mathrm{QW}$ structures for oriented charge transport have yet to be generated. Further systematic investigation of DJ phase perovskites may yield other promising alternative perovskites for elaboration into efficient Pb-free PSCs.

\subsection{D/3D hybrid perovskite heterojunction}

Limited by the large exciton binding energy and restricted charge transport across the spacer layers, the PCEs of current 2D PSCs under lower $n$-value phase still lag behind those of their state-of-the-art 3D analogs. Thus, aside from using the additive strategies described above to regulate the crystallization, film morphology and orientation of layered perovskites, spacer cations can be used as dopants to form 2D/3D hybrid heterojunctions that synergistically enhance device performance by suppressing ion migration and increasing long-term operational stability. The structure and optoelectronic properties of the $2 \mathrm{D} / 3 \mathrm{D}$ heterojunction depend on the chemical composition, ratio of its spacer cations, and the processing procedures that are used. It was shown that through introducing the long-chain $\mathrm{PEA}^{+}$into $\mathrm{MAPbI}_{3}$, the incorporated spacer cations were capable of interacting with inorganic perovskite slabs through ionic and hydrogen bonding, and induced the formation of $2 \mathrm{D} / 3 \mathrm{D}$ bulk heterojunction $[13,49]$. The spacer cations located at the lattice surface and grain boundaries effectively passivated the trap states, tightened the 3D crystal domains and inhibited phase transitions. However, an excess of 2D components in such hybrid 2D/3D perovskites led to restricted charge transport and a broad QW-thickness distribution, which caused charge accumulation and non-recombination loss.

Cheng and coworkers explored the effect of alkyl diammonium cation chain-length on the structural properties of such mixed-cation perovskites. It was found that longer alkyl diammonium cations promoted the formation of $2 \mathrm{D} / 3 \mathrm{D}$ hybrid structures, even at a relatively low concentration (Figure 7D). The use of ethane-1,2-diammonium $\left(\mathrm{EDA}^{2+}\right)$ as an organic cation and a low injection concentration prevented the formation of the $2 \mathrm{D}$ phase, and the resulting devices exhibited much improved environmental robustness with preserved efficiencies [112]. Fang et al. further incorporated $\mathrm{EDA}^{2+}$ into the $\mathrm{MA}_{0.7} \mathrm{FA}_{0.3} \mathrm{PbI}_{3}$ system, and the corresponding device had an improved PCE of 20.1\% [115]. Moreover, a series of alkyl diammonium cations were used to form functional 2D/3D hybrid heterojunction-containing perovskites. Tsang et al. revealed that the incorporation of $\mathrm{PDA}^{2+}$ into $\mathrm{Cs}_{0.15} \mathrm{FA}_{0.85} \mathrm{PbI}_{3}$ resulted in a perovskite with decreased crystallinity, and perovskite films with decreased hole mobility. As the $\mathrm{PDA}^{2+}$ content increased, it was observed that the trap-passivation effect increased in strength and competed with the emerging excitonic effect, resulting in a decrease in the relative dielectric constant, which generated concentration-dependent device efficiency [80]. 
A

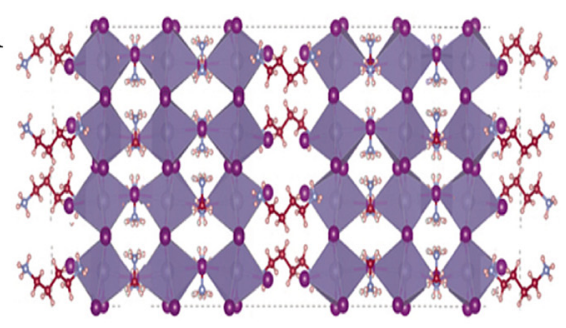

B

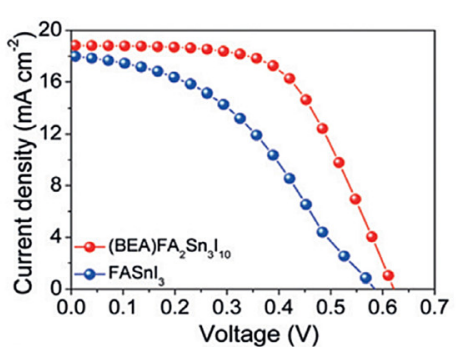

$\mathbf{E}$
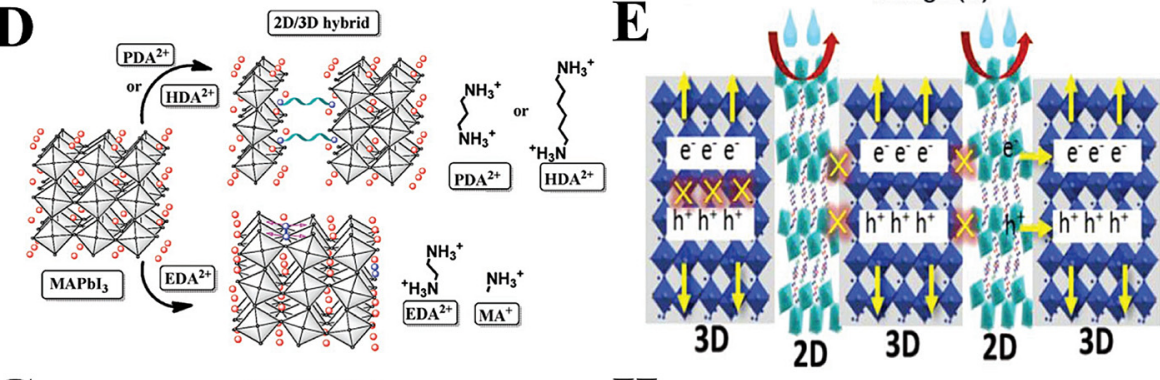

G

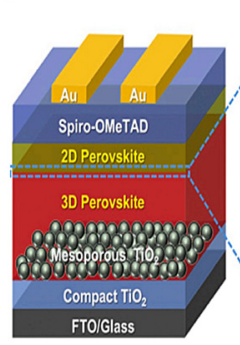

Lattice expansion

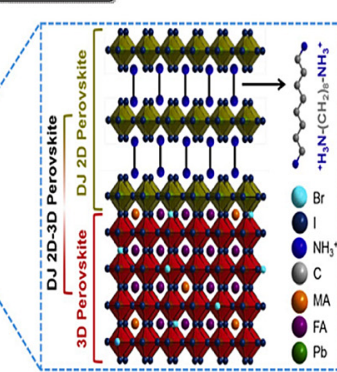

H
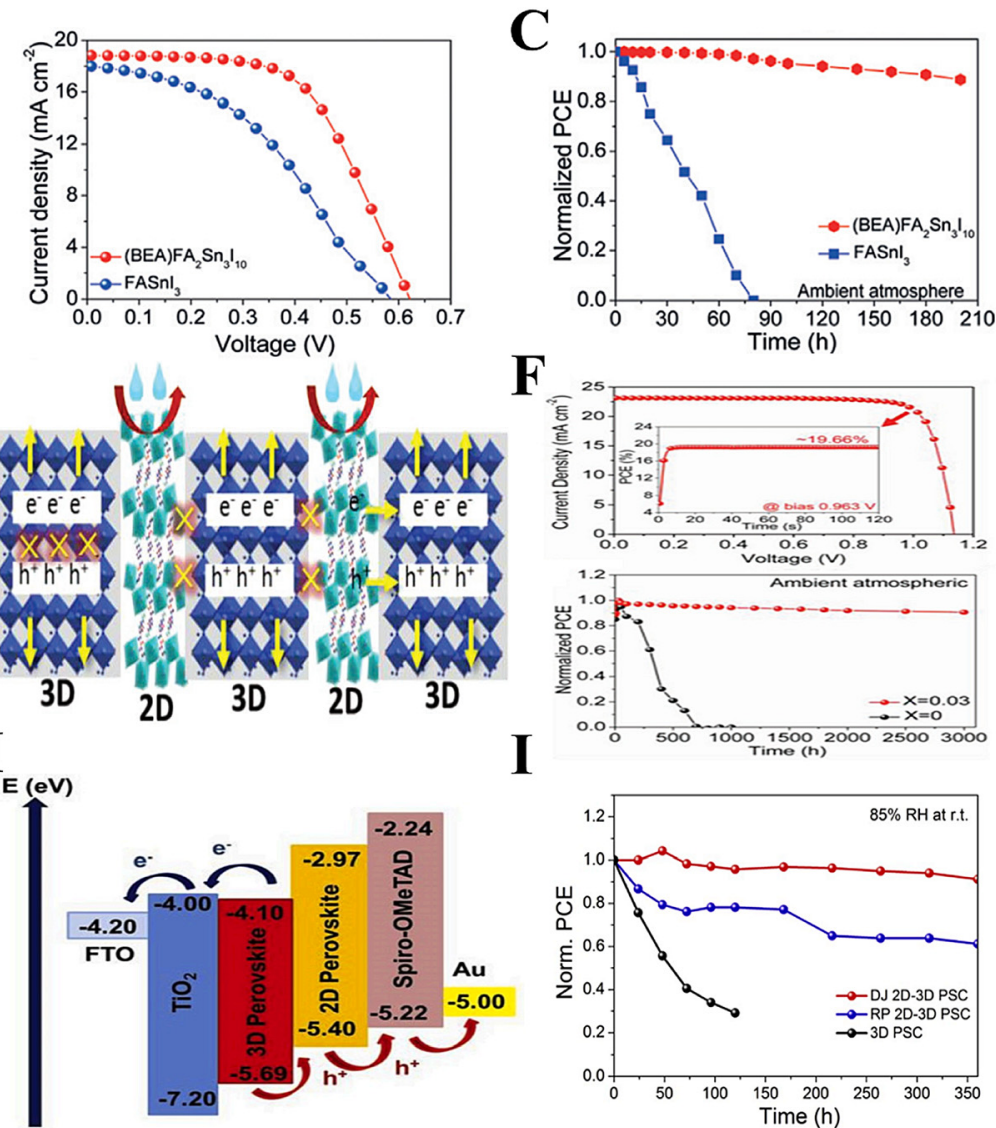

F
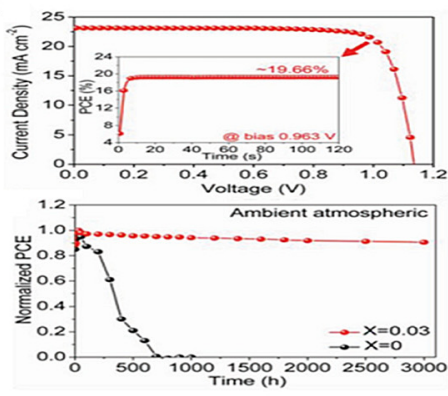

I

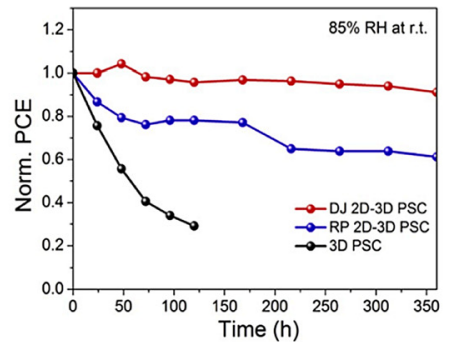

Figure 7: Applications in $\mathrm{Pb}$-free perovskite and 2D/3D hybrid hterojunction.

(A) Crystal structures of the 2D perovskite (BEA)(FA) ${ }_{2} \mathrm{Sn}_{3} \mathrm{l}_{10}$. (1.4-butanediamine was abbreviated as BEA here). (B) J-V characteristics of the champion-efficiency (BEA)FA $\mathrm{Sn}_{3} \mathrm{l}_{10}$ and FASnl ${ }_{3}$ devices. (C) Stability of (BEA)(FA) ${ }_{2} \mathrm{Sn}_{3} \mathrm{l}_{10}$ and $\mathrm{FASnl}_{3}$ devices under ambient atmosphere (ca. $40 \%$ humidity) without encapsulation. Reproduced with permission [76]. Copyright 2020, Wiley-VCH Publications. (D) The possible structural changes in $\mathrm{MAPbl}_{3}$ perovskite upon modification with different diammonium cations. Reproduced with permission [112]. Copyright 2017, Wiley-VCH Publications. (E) The schematic structure of $2 \mathrm{D} \mathrm{EDBEPbl}{ }_{4}$ layers interspersed 3D $\left(\mathrm{Cs}_{0.05}\left(\mathrm{FA}_{0.83} \mathrm{MA}_{0.17}\right)_{0.95} \mathrm{~Pb}\left(\mathrm{I}_{0.83} \mathrm{Br}_{0.17}\right)_{3}\right.$ perovskite with a phase-segregated vertical heterojunction (F) $J-V$ characteristic and steady-state output (inside) of the champion device (top) and module stability under $65 \%$ relative humidity conditions (bottom) of 2D/3D heterojunction and 3D PSCs. Reproduced with permission [81]. Copyright 2018, Wiley-VCH Publications. (G) Schematic illustration of the DJ 2D/3D perovskite hierarchical heterojunction based on ODAPbl $/$ $\left(\mathrm{FA}_{0.85} \mathrm{MA}_{0.15}\right) \mathrm{Pb}\left(\mathrm{I}_{0.85} \mathrm{Br}_{0.15}\right)_{3}$ and device architecture. $(\mathrm{H})$ Energy level diagram of the whole devices based on DJ phase 2D/3D perovskite. (I) Efficiency evolution of the 3D, RP phase 2D/3D, and DJ phase 2D/3D PSCs under constant humidity of $85 \%$ at room temperature. Reproduced with permission [84]. Copyright 2020, Elsevier Inc. Publications.

Similar to the findings from investigations of layered perovskites, 2D/3D hybrid perovskites formed via solution processing typically had variable QW thickness and phase dispersity, and this multiple QW nature and random distribution of QW thickness within bulk phases diminished carrier localization and charge transport [81]. To address this, Song et al. introduced phase-pure $\mathrm{C}_{6} \mathrm{H}_{18} \mathrm{~N}_{2} \mathrm{O}_{2} \mathrm{PbI}_{4}$ $\left(\mathrm{EDBEPbI}_{4}\right) 2 \mathrm{D}$ microcrystals into a precursor solution to control the self-assembly process and prevent the formation of multiple QW components. The presence of this $\mathrm{EDBEPbI}_{4}$ perovskite prolonged the crystallization process and vertically passivated the grain boundaries of the $3 \mathrm{D}$ $\left(\mathrm{Cs}_{0.05}\left(\mathrm{FA}_{0.83} \mathrm{MA}_{0.17}\right)_{0.95} \mathrm{~Pb}\left(\mathrm{I}_{0.83} \mathrm{Br}_{0.17}\right)_{3}\right)$ perovskite (Figure $\left.7 \mathrm{E}\right)$.
This yielded a film with improved surface quality and chargetransport properties, and the PCE of the corresponding device was $21.06 \%$ and its steady-state efficiency was $19.66 \%$. The phase-segregated 2D interlayer functioned as a protective layer against moisture and oxygen penetration and blocked ion migration, leading to the encapsulated module retaining $90 \%$ of its initial efficiency after a $3000 \mathrm{~h}$ aging test in air conditions (Figure 7F). In contrast to the formation of 2D phase perovskites by the insertion of long-chain cations into $3 \mathrm{D}$ perovskites, unusual one-dimensional (1D) quantum wires comprising edge-sharing inorganic octahedra surrounded by spacers were appeared in a 1,4-benzene diammonium (PhDA)-based system. Rather than being isolated by general 
spacer cations, the inorganic slabs in this case were isolated by the $1 \mathrm{D}$ perovskite, which led to the formation of unique low-dimensional perovskite phases [116]. The hydrophobic spacer cations in the two-sided domains of the $1 \mathrm{D}$ component protected the 3D perovskite from moisture and ion penetration, leading to enhanced device stability.

The introduction of the bulky ammonium cations into perovskite structure, terminating the integrity of the 3D framework, could therefore lead to the formation of layered perovskite structure. Building on the superior ambient stability of $2 \mathrm{D}$ counterpart, the $2 \mathrm{D} / 3 \mathrm{D}$ perovskite hierarchical heterojunction synergistically combines the favorable optoelectronic properties of the 3D perovskites through a cation-exchange process simply occurred on the top perovskite layer. The intercalation of the larger cations occurs to a limited depth, due to the discrepancy in their ionic radii. This results in deposition occur on the preformed 3D perovskite surface, followed by an in situ reaction with residual $\mathrm{PbI}_{2}$ forming the $2 \mathrm{D}$ capping layer via a post-growth process. The preserved structural integrity of the $2 \mathrm{D}$ and bulk phases benefits high efficiencies and stabilities of corresponding devices [117-119]. In addition to passivating surface traps and improving environmental resistance, wide-bandgap 2D perovskite capping layers generate gradient energy levels at contact interfaces, which aids selective charge transport, i.e., facilitating hole extraction from the underneath 3D perovskite while impeding electron transport.

Solution-processed polycrystalline perovskite films typically suffer from substantial structural disorder and interstitial defects and vacancies. To alleviate this, Alex et al. immersed annealed perovskite films into a series of diammonium iodide-based IPA solutions to highlight the importance of rational ligand design for efficient surface regulation and defect passivation. It was found that both $\mathrm{BDAI}_{2}$ - and $\mathrm{EDBEI}_{2}$-based films showed a greater thermodynamic tendency than other films to convert from a 3D to a 2D phase during the post-treatment process [120]. However, the activation energy of this phase conversion was closely related to the alkyl-chain length of the organic ligand. When the octadecyl ammonium (ODA) cation was incorporated as a spacer cation, it first needed to overcome the energy barrier to conversion from its energetically stable anti-state to its gauche conformation, before participating in the cation exchange. The required antigauche isomerization of this organic ligand increased the activation energy of the phase transition and hindered the formation of a 2D structure, but the surface passivation effect was retained. Consequently, the derived device had an efficiency of $17.60 \%$, compared to $14.64 \%$ for the device without ODA modification.
In a HDA-based 2D/3D planar stacking structure, a DJ phase 2D perovskite with $n=1$ on the surface functioned as a capping layer that protected the underlying 3D component from UV degradation [83]. The enhanced UV durability of this DJ phase 2D/3D structure largely originated from the strong electrostatic interaction between diammonium spacer cations and inorganic slabs in the stacking direction, which created a robust architecture. The 2D capping layer also passivated the surface defects and suppressed charge recombination at contact interfaces, which resulted in a PCE improvement in the corresponding device, from $19.22 \%$ in the pristine $3 \mathrm{D}$ device to $20.31 \%$ in the $2 \mathrm{D} / 3 \mathrm{D}$ device, which also had better moisture and thermal stabilities. $\mathrm{Li}$ et al. spin-coated $\mathrm{ODAI}_{2}$-containing IPA solution onto annealed 3D perovskite films, and subjected these to further thermal treatment to form a DJ phase 2D/3D hierarchical heterojunction, which was elaborated into a device with an efficiency of $21.6 \%$ (Figure 7G) [84]. The highest occupied molecular orbital (HOMO) level of the 2D/3D perovskite was aligned with its hole transport layer (HTL), which led to fast interfacial hole transport and extraction. The high lowest unoccupied molecular orbital (LUMO) level of the upper 2D perovskite limited the back transport of electrons from its 3D counterpart to the HTL, which decreased non-radiative recombination (Figure $7 \mathrm{H}$ ). Moreover, the structural stability advantages of this DJ phase 2D perovskite resulted in the corresponding 2D/3D device having good environmental durability under harsh stresses, such that it retained $90 \%$ of its original PCE after exposure to $85 \%$ humidity for $360 \mathrm{~h}$. In contrast, the widely investigated RP phase PEA-based 2D/3D cells suffered a 38\% PCE loss under the same aging period, indicating the superior practical potential of DJ phase hierarchical heterojunctions (Figure 7I).

Inspired by the previous experience from 2D perovskite research, the rational tailoring of spacer ligands could enable further tuning of the electronic structures, crystal orientations and optoelectronic properties of 2D perovskite capping layer. By combining the excellent stability of 2D perovskites with the desirable photovoltaic properties of 3D components, the well-defined 2D/3D perovskite hybrid heterojunction represents a promising strategy to balance the trade-off between efficiency and stability and push forward the timelycommercialization of PSCs.

\section{Conclusion and further outlook}

The bulky hydrophobic spacer cations and inhabited ion migration within $2 \mathrm{D}$ perovskites endow them a better 
environmental stability against that of 3D counterparts. Among them, the DJ phase 2D perovskites occupy a shorter I...I interlayer distances, about 4.0-4.2 $\AA$ and narrower optical bandgaps compared to their RP phase analogs, which provides a new category for further developing the stable and efficient perovskite photovoltaic materials. The basic understandings of structure-property relationship, the improvements in synthesize and deposition skills and advancements in relevant characterization technologies would continuously push forward the development in device performance. However, some key issues and relevant crystallization and aging mechanisms still face the challenges and need the further explorations. Here, we summarize the remained barriers in the current DJ phase 2D perovskites to search for the future prospects and developing directions, as shown below.

\subsection{Access to pure, high $n$-value phases}

DJ phase 2D perovskites processed using traditional solution methods inevitably suffer from phase diversity and random QW distribution, which largely precludes improvements in device performance. The presence of solvent-incorporating intermediate phases and the strong electrostatic affinity between the terminal $-\mathrm{NH}_{3}{ }^{+}$groups of spacer cations and inorganic slabs mean that it is thermodynamically difficult to control phase purity during the self-assembly process. DJ phase 2D perovskite structures generally consist of high $n$-value phases present within low $n$-value components, and the thickness of QWs gradually increases through a substrate to the film surface, similar to what is observed in RP phase perovskites. Therefore, socalled layered perovskites are more inclined to mixed structures, as they contain multiple distributions of QWs with various $n$-values. It has been shown that multiple phases in films with compositions of $n>1$ form a cascade band-aligned structure and regulate device performance via a consecutive photo-induced charge separation and transfer process that funnels carriers to opposite electrodes [60]. However, heterogeneous QW components and arrangements lead to anisotropic charge transport and impede the efficiency of charge separation and extraction. The wide range of phase compositions in 2D perovskites, each with varying degrees of quantum confinement, results in carrier localization and decreased mobility. In addition, the random distribution of multiple QWs in 2D perovskites can adversely affect the stability of derived devices. In pure-phase films, the perovskite frameworks are encapsulated by the hydrophobic spacer layers, whereas in phase-diverse films the presence of higher $n$-value phases decreases hydrophobicity and destroys structural stability.

As for the field of crystal synthesis, the pure multilayer DJ phase perovskite with higher-n number $(n \geq 5)$ are still facing the challenges. The strong hydrogen bonds between diammonium cations and inorganic layers lead to poor solubility in $n=1$ forms, which rapidly precipitate out of solution and suppress the formation of higher $n$-value phases. Consequently, the formation of phase-pure layered perovskites with higher $n$-values $(n>4)$ is thermodynamically disfavored, and thus they are difficult to synthesize and detect. It is therefore encouraging that Kanatzidis and coworkers combined the conventional HI solution method with solid-state grinding to obtain a series of DJ phase compounds with varied ligand chain-lengths and QW thicknesses [88]. Another advance was the preparation of a pure-phase film by using an ionic liquid (e.g., methylamine acetate) as a solvent, combined with the short-chain ligands (PDA and BDA), to afford DJ phase perovskite films with uniform QW distributions [94]. It is critical that new synthetic and deposition technologies are developed to enable the formation of thermodynamically phase-stable 2D components via solution processes. This will allow unambiguous determination of material properties, and thus promote DJ phase 2D perovskites as competitive candidates for commercial applications.

\subsection{Ligand design}

The spacer interlayer between metal-halide inorganic slabs within DJ phase perovskites contains a single diammonium cation per unit, which results in stacks with a highly symmetrical alignment or a minor displacement, depending on the steric requirements of the spacer cations. The structures of organic ligands in DJ phase perovskites differ from those used in RP phase perovskites, which leads to the closer and more uniform stacking of inorganic slabs in layered structures. The structural degrees of freedom and the orbital overlap within the spacer layer depend on the spatial arrangement of spacer cations and the interaction between adjacent moieties [22, 78]. On the synthetic chemistry front, the abundance of organic ligands provides a large space to explore for the new archetype of lowdimensional photovoltaic materials and provide huge potential for novel hybrid combinations with perovskite layers [21, 54]. The tailoring of ligand structures at the molecular level combined with the exploration of alternative layered perovskite formulations will lead to new classes of high-performance low-dimensional PSCs. However, the ligand library in the case of integrated organic 
spacer cations used in DJ phase perovskites is limited, especially for the 2D/3D hybrid perovskite system.

Functionalization on the organic side and tailoring of bifunctional or multifunctional organic ligands to induce the controlled self-assembly process and improve crystallization and nucleation behavior of $\mathrm{DJ}$ phase perovskites is still an urgent research direction needed to be resolved. The synthesis of novel ligand materials will help to overcome the current efficiency bottleneck in 2D PSCs, and afford devices with superior long-term stability. New techniques for the fluorination of aromatic organic-spacer cations have been used to improve charge transport properties in RP phase 2D perovskites. A detailed investigation of fluorination- or functional side-chain substituentinduced changes in structural and intermolecular electronic coupling between neighboring organic cations could lead to the incorporation of a wide variety of bifunctional organic molecules in layered perovskites, and improve the photovoltaic performance of the corresponding PSCs. Further exploration is likely to advance the development of this field and unlock the new territory of efficient DJ phase 2D perovskites.

\subsection{Develop the alternative charge transport materials (CTMs)}

The high energy level of the conduction band in typical 2D perovskites means that they generally suffer from a large energy barrier at contact interfaces with commonly used electron transport layers. This poor energy alignment at electron-extraction interfaces restricts charge separation and extraction, and electronic selectivity, by blocking back-transfer of charge, leading to large recombination losses in devices. On the other hand, for the alternative CTMs employed in DJ phase PSCs, the degree of lattice matching between the 2D perovskites and the bottom CTMs is closely related to the crystalline orientation. Better lattice matching will reduce the distortion energy of the contact interface and improve the vertical arrangement of QWs [61]. Unfortunately, the choice of CTMs, that are appropriate to the DJ phase PSCs, is also limited. The combination of efficient and stable dopant-free hole transport materials could largely prevent CTM-induced device degradation, and further improve device longevities under various harsh environmental conditions [121]. The delineation of how the physical and chemical properties of CTMs affect crystallization dynamics, thickness distribution and orientation of QWs, and related optoelectronic properties in DJ phase perovskites is still lacking. Such shortage may hinder the further development of DJ phase PSCs.
Self-assembled monolayers (SAMs) have a unique range of abilities, as they are able to passivate traps, control crystallinity, adjust band energies and modify charge dynamics, and thus SAMs have been used for interfacial decoration. SAM-based regulation of bottom interfacial contact may enable the optimization of surface energy and thus induce preferred crystallization kinetics during the formation of DJ phase perovskites, which would offer an excellent opportunity to tune the interfacial properties of corresponding devices. Therefore, the development of alternative interfacial modification strategies, and interfacial materials with good optical, electrical and electronic properties, is crucial for the fabrication of more stable and efficient layered PSCs.

\subsection{Determination of degradation mechanisms}

It is widely accepted that the $\mathrm{DJ}$ phase $2 \mathrm{D}$ perovskites have greater structural stability under various harsh environmental stresses than their 3D and RP phase counterparts. The main reasons for this superior stability can be concluded into four aspects, including (1) the strong hydrophobicity of spacer cations, which isolates the absorbing layer from ambient conditions; (2) the robust hydrogen-bonding interaction between diammonium cations and inorganic slabs, which stabilizes the perovskite structure; (3) the compact crystal stacking, which generates high film crystallinity; and (4) the suppression of trap density in films, which hinders the penetration of moisture and oxygen in ambient conditions [34]. However, the microscopic degradation mechanisms of DJ phase PSCs under long-term operational conditions have yet to be explored, including the aspects of chemical composition degradation, exciton evolution dependent on condensed state structure, the attenuation of optoelectronic properties.

In a 2D/3D hierarchical structure, a dynamic evolution of composition was previously observed in humidity conditions, whereby high $n$-value phases reacted with $\mathrm{H}_{2} \mathrm{O}$ to form $\mathrm{MAPbI}_{3} \cdot \mathrm{H}_{2} \mathrm{O}$ and smaller $n$-value components, thus enhancing the surface hydrophobicity [122]. Under thermal stress, an inverse process occurred, in which higher $n$-value phases were formed as MA species were continuously released into an upper 2D perovskite, which acted as a buffer layer that suppressed ion migration at elevated temperatures. However, the effects of environmental factors, component evolution and interfacial contact on the dynamic evolution of DJ phase PSCs under long-term exposure remain unknown. The degradation mechanisms 
of perovskites must therefore be explored to delineate the relationship between environmental factors, QW components, and charge distribution and accumulation over time, to allow the rational selection of materials to form highly stable 2D perovskites.

The development of advanced deposition techniques and characterization methods will assist in the detailed identification of the aging behaviors of layered perovskites during degradation, to guide further rational selection of appropriate interfacial materials, and preparation and encapsulation techniques. Given the stability advantages of DJ phase perovskites, access to well-defined combinations of ligand materials, deposition-processing methods and device-architecture combinations should enable the fabrication of devices that meet the IEC61215:2016 stability standards [123].

\subsection{Reverse phase distribution}

Solution-processed 2D perovskites generally comprise a multiphase distribution, with $n$-values increasing from the bottom to the top of a substrate, and have different stoichiometric ratios of components from those in their precursor solutions. However, this internal band alignment does not facilitate charge collection within conventional $n$ $i-p$ devices. Methods for the regulation of the phase alignment direction according to device structure are therefore needed to enable the development of high-performance layered PCEs. Anti-solvent engineering and functional spacer cations have been used to control the phase distribution and flip the band alignment of RP phase components. For example, Wang et al. used ethyl acetate as an anti-solvent to accelerate surface-level crystal formation in RP phase perovskites, which afforded control of crystal growth directions [124]. Zhao et al. reported a similar effect by using cyclohexane methylamine as a spacer cation to provide altered ligand chemistry. The resulting deposited RP phase perovskites exhibited a reverse-graded phase distribution in a conventional device; the smaller $n$-value phases at the surface facilitated charge transport, hindered water permeation and therefore enhanced long-term device stability [125]. This approach could be explored in DJ phase perovskites to enable phase alignment and flattened energy landscapes to be obtained for efficient charge transport.

Currently, the highest reported efficiency for the DJ phase PSCs is more than $18 \%$, and this was prepared using a simple solution process under ambient air conditions, indicating the practical potential of these materials $[34,75]$.
Increasing numbers of studies have reported 2D/3D hybrid perovskite-based devices that exhibited simultaneous enhancements in stability and efficiency, with 2D components serving as passivation agents or capping layers [126-128]. The wide processing window of these unique photovoltaic materials will trigger more academic and industrial interest in combining them with various printing technologies for scaled-up fabrication or use in tandem configuration devices. It is reasonable to predict great progress in this active research field in the near future, ultimately leading to the commercialization and mass production of cost-efficient and green DJ phase perovskite based photovoltaic devices.

Author contributions: All the authors have accepted responsibility for the entire content of this submitted manuscript and approved submission.

Research funding: The work was financially supported by the Guangdong Major Project of Basic and Applied Basic Research (No. 2019B030302007); Guangdong Basic and Applied Basic Research Foundation for Distinguished Young Scholar (No. 2021B1515020028); the Ministry of Science and Technology (Nos. 2017YFA0206600 and 2019YFA0705900); the Natural Science Foundation of China (Nos. 51973063, 91733302, and 51803060); the Science and Technology Program of Guangdong Province, China (No. 2018A030313045); the Science and Technology Program of Guangzhou, China (No. 201904010147).

Conflict of interest statement: The authors declare no conflicts of interest regarding this article.

\section{References}

[1] G. Xing, N. Mathews, S. Sun, et al., “Long-range balanced electronand hole-transport lengths in organic-inorganic $\mathrm{CH}_{3} \mathrm{NH}_{3} \mathrm{Pbl}_{3}$," Science, vol. 342, pp. 344-347, 2013.

[2] J. Burschka, N. Pellet, S.-J. Moon, et al., "Sequential deposition as a route to high-performance perovskite-sensitized solar cells," Nature, vol. 499, pp. 316-319, 2013.

[3] S. Sun, T. Salim, N. Mathews, et al., "The origin of high efficiency in low-temperature solution-processable bilayer organometal halide hybrid solar cells," Energy Environ. Sci., vol. 7, pp. 399-407, 2014.

[4] D. Shi, V. Adinolfi, R. Comin, et al., "Low trap-state density and long carrier diffusion in organolead trihalide perovskite single crystals," Science, vol. 347, pp. 519-522, 2015.

[5] N. J. Jeon, J. H. Noh, W. S. Yang, et al., “Compositional engineering of perovskite materials for high-performance solar cells," Nature, vol. 517, pp. 476-480, 2015.

[6] A. Kojima, K. Teshima, Y. Shirai, and T. Miyasaka, “Organometal halide perovskites as visible-light sensitizers for photovoltaic cells," J. Am. Chem. Soc., vol. 131, pp. 6050-6051, 2009. 
[7] National Renewable Energy Laboratory, "Best research-cell efficiency chart,” Available at: www.nrel.gov/pv/cell-efficiency. html.

[8] J. You and Y. Yang, "Make perovskite solar cells stable," Nature, vol. 544, pp. 155-156, 2017.

[9] A. Buin, P. Pietsch, J. Xu, et al., "Materials processing routes to trap-free halide perovskites," Nano Lett., vol. 14, pp. 6281-6286, 2014.

[10] D. Bryant, N. Aristidou, S. Pont, et al., "Light and oxygen induced degradation limits the operational stability of methylammonium lead triiodide perovskite solar cells," Energy Environ. Sci., vol. 9, pp. 1655-1660, 2016.

[11] M. Grätzel, "The rise of highly efficient and stable perovskite solar cells," Acc. Chem. Res., vol. 50, pp. 487-491, 2017.

[12] T. A. Berhe, W.-N. Su, C.-H. Chen, et al., "Organometal halide perovskite solar cells: degradation and stability," Energy Environ. Sci., vol. 9, pp. 323-356, 2016.

[13] I. C. Smith, E. T. Hoke, D. Solis-Ibarra, M. D. McGehee, and H. I. Karunadasa, "A layered hybrid perovskite solar-cell absorber with enhanced moisture stability," Angew. Chem. Int. Ed., vol. 53, pp. 11232-11235, 2014.

[14] D. H. Cao, C. C. Stoumpos, O. K. Farha, J. T. Hupp, and M. G. Kanatzidis, "2D homologous perovskites as lightabsorbing materials for solar cell applications," J. Am. Chem. Soc., vol. 137, pp. 7843-7850, 2015.

[15] G. Grancini and M. K. Nazeeruddin, "Dimensional tailoring of hybrid perovskites for photovoltaics," Nat. Rev. Mater., vol. 4, pp. 4-22, 2018.

[16] D. B. Mitzi, “Organic-inorganic perovskites containing trivalent metal halide layers: the templating influence of the organic cation layer," Inorg. Chem., vol. 39, pp. 6107-6113, 2000.

[17] C. C. Stoumpos, D. H. Cao, D. J. Clark, et al., “Ruddlesdenpopper hybrid lead iodide perovskite 2D homologous semiconductors," Chem. Mater., vol. 28, pp. 2852-2867, 2016.

[18] L. Etgar, "The merit of perovskite's dimensionality; can this replace the 3D halide perovskite?," Energy Environ. Sci., vol. 11, pp. 234-242, 2018.

[19] J.-P. Correa-Baena, M. Saliba, T. Buonassisi, et al., "Promises and challenges of perovskite solar cells," Science, vol. 358, pp. 739-744, 2017.

[20] G. Kieslich, S. Sun, and A. K. Cheetham, "Solid-state principles applied to organic-inorganic perovskites: new tricks for an old dog," Chem. Sci., vol. 5, pp. 4712-4715, 2014.

[21] L. Mao, C. C. Stoumpos, and M. G. Kanatzidis, "Two-dimensional hybrid halide perovskites: principles and promises," J. Am. Chem. Soc., vol. 141, pp. 1171-1190, 2018.

[22] L. Mao, W. Ke, L. Pedesseau, et al., "Hybrid dion-jacobson 2D lead iodide perovskites,” J. Am. Chem. Soc., vol. 140, pp. 3775-3783, 2018.

[23] H. Tsai, W. Nie, J.-C. Blancon, et al., "High-efficiency twodimensional Ruddlesden-Popper perovskite solar cells," Nature, vol. 536, pp. 312-316, 2016.

[24] G. Wu, X. Li, J. Zhou, et al., "Fine multi-phase Alignments in 2D perovskite solar cells with efficiency over $17 \%$ via slow postannealing," Adv. Mater., vol. 31, p. 1903889, 2019.

[25] C. M. M. Soe, W. Nie, C. C. Stoumpos, et al., "Understanding film formation morphology and orientation in high member $2 \mathrm{D}$ ruddlesden-popper perovskites for high-efficiency solar cells," Adv. Energy Mater., vol. 8, p. 1700979, 2018.
[26] X. Zhang, R. Munir, Z. Xu, et al., "Phase transition control for high performance ruddlesden-popper perovskite solar cells," Adv. Mater., vol. 30, p. 1707166, 2018.

[27] X. Zhang, X. Ren, B. Liu, et al., "Stable high efficiency twodimensional perovskite solar cells via cesium doping," Energy Environ. Sci., vol. 10, pp. 2095-2102, 2017.

[28] C. Liang, H. Gu, Y. Xia, et al., "Two-dimensional RuddlesdenPopper layered perovskite solar cells based on phase-pure thin films," Nat. Energy, vol. 6, pp. 38-45, 2020.

[29] H. Ren, S. Yu, L. Chao, et al., "Efficient and stable RuddlesdenPopper perovskite solar cell with tailored interlayer molecular interaction,” Nat. Photonics, vol. 14, pp. 154-163, 2020.

[30] J. Zhang, J. Qin, M. Wang, et al., "Uniform permutation of quasi2D perovskites by vacuum poling for efficient, high-fill-factor solar cells," Joule, vol. 3, pp. 3061-3071, 2019.

[31] X. Li, G. Wu, M. Wang, et al., "Water-assisted Crystal Growth in Quasi-2D Perovskites with enhanced charge transport and photovoltaic performance," Adv.Energy Mater., vol. 10, p. 2001832, 2020.

[32] R. Yang, R. Li, Y. Cao, et al., "Oriented quasi-2D perovskites for high performance optoelectronic devices," Adv. Mater., vol. 30, p. $1804771,2018$.

[33] G. Wu, T. Yang, X. Li, et al., "Molecular engineering for twodimensional perovskites with photovoltaic efficiency exceeding 18\%," Matter, vol. 4, pp. 582-599, 2021.

[34] T. Niu, H. Ren, B. Wu, et al., "Reduced-dimensional perovskite enabled by organic diamine for efficient photovoltaics," J. Phys. Chem. Lett., vol. 10, pp. 2349-2356, 2019.

[35] S. Ahmad, P. Fu, S. Yu, et al., "Dion-Jacobson phase 2D layered perovskites for solar cells with ultrahigh stability," Joule, vol. 3, pp. 794-806, 2019.

[36] C. Ma, D. Shen, T.-W. Ng, M.-F. Lo, and C.-S. Lee, "2D perovskites with short interlayer distance for high-performance solar cell application," Adv. Mater., vol. 30, p. 1800710, 2018.

[37] P. Huang, S. Kazim, M. Wang, and S. Ahmad, "Toward phase stability: Dion-Jacobson layered perovskite for solar cells," ACS Energy Lett., vol. 4, pp. 2960-2974, 2019.

[38] C. C. Stoumpos, C. M. M. Soe, H. Tsai, et al., "High members of the $2 \mathrm{D}$ ruddlesden-popper halide perovskites: synthesis, optical properties, and solar cells of $\left(\mathrm{CH}_{3}\left(\mathrm{CH}_{2}\right)_{3} \mathrm{NH}_{3}\right)_{2}\left(\mathrm{CH}_{3} \mathrm{NH}_{3}\right)_{4} \mathrm{~Pb}_{5} \mathrm{I}_{16}$," Chem, vol. 2, pp. 427-440, 2017.

[39] S. N. Ruddlesden and P. Popper, "New compounds of the $\mathrm{K}_{2} \mathrm{NiF}_{4}$ type,” Acta Crystallogr., vol. 10, pp. 538-539, 1957.

[40] A. J. Jacobson, J. W. Johnson, and J. T. Lewandowski, “Interlayer chemistry between thick transition-metal oxide layers: synthesis and intercalation reactions of $\mathrm{K}\left[\mathrm{Ca}_{2} \mathrm{Na}_{n-3} \mathrm{Nb}_{n} \mathrm{O}_{3 \mathrm{n}+1}\right](3 \leq \mathrm{n} \leq 7)$," Inorg. Chem., vol. 24, pp. 3727-3729, 1985.

[41] C. M. M. Soe, C. C. Stoumpos, M. Kepenekian, et al., "New yype of $2 \mathrm{D}$ perovskites with alternating cations in the interlayer space, $\left(\mathrm{C}\left(\mathrm{NH}_{2}\right)_{3}\right)\left(\mathrm{CH}_{3} \mathrm{NH}_{3}\right)_{\mathrm{n}} \mathrm{Pb}_{\mathrm{n}} \mathrm{I}_{3 \mathrm{n}+1}$ : structure, properties, and photovoltaic performance," J. Am. Chem. Soc., vol. 139, pp. 16297-16309, 2017.

[42] K. R. Kendall, C. Navas, J. K. Thomas, and H.-C. Z. Loye, "Recent developments in oxide lon conductors: aurivillius phases," Chem. Mater., vol. 8, pp. 642-649, 1996.

[43] C. H. Hervoches and P. Lightfoot, "A variable-temperature powder neutron diffraction study of ferroelectric $\mathrm{Bi}_{4} \mathrm{Ti}_{3} \mathrm{O}_{12}$," Chem. Mater., vol. 11, pp. 3359-3364, 1999.

[44] T. Hu, M. D. Smith, E. R. Dohner, et al., "Mechanism for broadband white-Light emission from two-dimensional (110) 
hybrid perovskites," J. Phys. Chem. Lett., vol. 7, pp. 2258-2263, 2016.

[45] B. Saparov and D. B. Mitzi, "Organic-inorganic perovskites: structural versatility for functional materials design," Chem. Rev., vol. 116, pp. 4558-4596, 2016.

[46] F. Zhang, H. Lu, J. Tong, J. J. Berry, M. C. Beard, and K. Zhu, "Advances in two-dimensional organic-inorganic hybrid perovskites,” Energy Environ. Sci., vol. 13, pp. 1154-1186, 2020.

[47] Y. Zhang, P. Wang, M.-C. Tang, et al., "Dynamical transformation of two-dimensional perovskites with alternating cations in the interlayer space for high-performance photovoltaics," J. Am. Chem. Soc., vol. 141, pp. 2684-2694, 2019.

[48] T. Luo, Y. Zhang, Z. Xu, et al., "Compositional control in 2D perovskites with alternating cations in the interlayer space for photovoltaics with efficiency over 18\%," Adv. Mater., vol. 31, p. 1903848, 2019.

[49] L. N. Quan, M. Yuan, R. Comin, et al., "Ligand-stabilized reduceddimensionality perovskites," J. Am. Chem. Soc., vol. 138, pp. 2649-2655, 2016.

[50] M. Sato, J. Abo, T. Jin, and M. Ohta, "Structure determination of $\mathrm{KLaNb}_{2} \mathrm{O}_{7}$ exhibiting ion exchange ability by $\mathrm{X}$-ray powder diffraction," Solid State Ionics, vol. 51, pp. 85-89, 1992.

[51] N. Kumada, N. Kinomura, and A. W. Sleight, "CsLaNb $2 \mathrm{O}_{7}$, , Acta Crystallogr. Sect. C Cryst. Struct. Commun., vol. 52, pp. 1063-1065, 1996.

[52] X. Hong, T. Ishihara, and A. V. Nurmikko, "Dielectric confinement effect on excitons inPbl4-based layered semiconductors," Phys. Rev. B, vol. 45, pp. 6961-6964, 1992.

[53] R. L. Milot, R. J. Sutton, G. E. Eperon, et al., "Charge-carrier dynamics in 2D hybrid metal-halide perovskites," Nano Lett., vol. 16, pp. 7001-7007, 2016.

[54] D. Thrithamarassery Gangadharan and D. Ma, "Searching for stability at lower dimensions: current trends and future prospects of layered perovskite solar cells," Energy Environ. Sci., vol. 12, pp. 2860-2889, 2019.

[55] E. R. Dohner, A. Jaffe, L. R. Bradshaw, and H. I. Karunadasa, "Intrinsic white-light emission from layered hybrid perovskites," J. Am. Chem. Soc., vol. 136, pp. 13154-13157, 2014.

[56] D. Sapori, M. Kepenekian, L. Pedesseau, C. Katan, and J. Even, "Quantum confinement and dielectric profiles of colloidal nanoplatelets of halide inorganic and hybrid organic-inorganic perovskites," Nanoscale, vol. 8, pp. 6369-6378, 2016.

[57] D. Varsano, G. Giorgi, K. Yamashita, and M. Palummo, "Role of quantum-confinement in anatase nanosheets," J. Phys. Chem. Lett., vol. 8, pp. 3867-3873, 2017.

[58] A. Z. Chen, M. Shiu, J. H. Ma, et al., "Origin of vertical orientation in two-dimensional metal halide perovskites and its effect on photovoltaic performance,” Nat. Commun., vol. 9, p. 1336, 2018.

[59] X. Gao, X. Zhang, W. Yin, et al., "Ruddlesden-popper perovskites: synthesis and optical properties for optoelectronic applications," Adv. Sci., vol. 6, p. 1900941, 2019.

[60] J. Liu, J. Leng, K. Wu, J. Zhang, and S. Jin, "Observation of internal photoinduced electron and hole separation in hybrid twodimentional perovskite films," J. Am. Chem. Soc., vol. 139, pp. 1432-1435, 2017.

[61] Y. Xu, M. Wang, Y. Lei, Z. Ci, and Z. Jin, “Crystallization kinetics in 2D perovskite solar cells," Adv. Energy Mater., vol. 10, p. 2002558, 2020.

[62] S. Sourisseau, N. Louvain, W. Bi, et al., "Reduced band gap hybrid perovskites resulting from combined hydrogen and halogen bonding at the organic-inorganic interface," Chem. Mater., vol. 19, pp. 600-607, 2007.

[63] Y. Zheng, T. Niu, J. Qiu, et al., "Oriented and uniform distribution of dion-jacobson phase perovskites controlled by quantum well barrier thickness," Solar RRL, vol. 3, p. 1900090, 2019.

[64] X. Li, W. Ke, B. Traoré, et al., "Two-dimensional Dion-Jacobson hybrid lead iodide perovskites with aromatic diammonium cations," J. Am. Chem. Soc., vol. 141, pp. 12880-12890, 2019.

[65] W. Ke, L. Mao, C. C. Stoumpos, et al., "Compositional and solvent engineering in Dion-Jacobson 2D perovskites boosts solar cell efficiency and stability," Adv. Energy Mater., vol. 9, p. 1803384, 2019.

[66] H. Wu, X. Lian, S. Tian, et al., “Additive-sssisted hot-casting free fabrication of Dion-Jacobson 2D perovskite solar cell with efficiency Bbeyond 16\%," Solar RRL, vol. 4, p. 2000087, 2020.

[67] M. Chen, M.-G. Ju, M. Hu, et al., "Lead-free Dion-Jacobson tin halide perovskites for photovoltaics," ACS Energy Lett., vol. 4, pp. 276-277, 2019.

[68] D. Lu, G. Lv, Z. Xu, Y. Dong, X. Ji, and Y. Liu, “Thiophene-based two-dimensional Dion-Jacobson perovskite solar cells with over 15\% efficiency," J. Am. Chem. Soc., vol. 142, pp. 11114-11122, 2020.

[69] W. Zhao, Q. Dong, J. Zhang, et al., "Asymmetric alkyl diamine based Dion-Jacobson low-dimensional perovskite solar cells with efficiency exceeding 15\%," J. Mater. Chem. A, vol. 8, pp. 9919-9926, 2020.

[70] L. Cheng, Z. Liu, S. Li, et al., "Highly thermostable and efficient formamidinium-based low-dimensional perovskite solar cells," Angew. Chem. Int. Ed., vol. 60, pp. 856-864, 2021.

[71] J. Wang, D. Lin, Y. Chen, et al., "Suppressing the excessive solvated phase for Dion-Jacobson perovskites with improved crystallinity and vertical orientation," Solar RRL, vol. 4, p. 2000371, 2020.

[72] M. Safdari, P. H. Svensson, M. T. Hoang, I. Oh, L. Kloo, and J. M. Gardner, "Layered 2D alkyldiammonium lead iodide perovskites: synthesis, characterization, and use in solar cells," J. Mater. Chem. A, vol. 4, pp. 15638-15646, 2016.

[73] M. Safdari, D. Phuyal, B. Philippe, et al., "Impact of synthetic routes on the structural and physical properties of butyl-1,4diammonium lead iodide semiconductors," J. Mater. Chem. A, vol. 5, pp. 11730-11738, 2017.

[74] F. Li, J. Zhang, S. Jo, et al., "Vertical orientated Dion-Jacobson quasi-2D perovskite film with improved photovoltaic performance and stability," Small Methods, vol. 4, p. 1900831, 2019.

[75] X. Zhao, T. Liu, A. B. Kaplan, C. Yao, and Y.-L. Loo, “Accessing highly oriented two-dimensional perovskite films via solventvapor annealing for efficient and stable solar cells," Nano Lett., vol. 20, pp. 8880-8889, 2020.

[76] P. Li, X. Liu, Y. Zhang, et al., "Low-dimensional Dion-Jacobsonphase lead-free perovskites for high-performance photovoltaics with improved stability," Angew. Chem. Int. Ed., vol. 59, pp. 6909-6914, 2020.

[77] H. Wang, Z. Qin, J. Xie, et al., "Efficient slantwise aligned DionJacobson phase perovskite solar cells based on trans-1,4cyclohexanediamine," Small, vol. 16, p. 2003098, 2020.

[78] Y. Li, J. V. Milić, A. Ummadisingu, et al., "Bifunctional organic spacers for formamidinium-based hybrid Dion-Jacobson twodimensional perovskite solar cells," Nano Lett., vol. 19, pp. 150-157, 2019. 
[79] B.-E. Cohen, Y. Li, Q. Meng, and L. Etgar, “Dion-Jacobson twodimensional perovskite solar cells based on benzene dimethanammonium cation," Nano Lett., vol. 19, pp. 2588-2597, 2019.

[80] X. Xu, Y.-M. Xie, Y. Ma, et al., "The Role of diammonium cation on the structural and optoelectronic properties in 3D cesiumformamidinium mixed-cation Perovskite solar cells," Solar RRL, vol. 3, p. 1900140, 2019.

[81] P. Li, Y. Zhang, C. Liang, et al., "Phase pure 2D perovskite for high-performance 2D-3D heterostructured perovskite solar cells," Adv. Mater., vol. 30, p. 1805323, 2018.

[82] W. Ke, C. Chen, I. Spanopoulos, et al., "Narrow-bandgap mixed lead/tin-based 2D Dion-Jacobson perovskites boost the performance of solar cells," J. Am. Chem. Soc., vol. 142, pp. 15049-15057, 2020.

[83] Y. Lv, H. Ma, Y. Yin, et al., “ $\left[\mathrm{NH}_{3}\left(\mathrm{CH}_{2}\right)_{6} \mathrm{NH}_{3}\right] \mathrm{Pbl} \mathrm{Pl}_{4}$ as Dion-Jacobson phase bifunctional capping layer for $2 \mathrm{D} / 3 \mathrm{D}$ perovskite solar cells with high efficiency and excellent UV stability," J. Mater. Chem. A, vol. 8, pp. 10283-10290, 2020.

[84] X. Jiang, J. Zhang, S. Ahmad, et al., "Dion-Jacobson 2D-3D perovskite solar cells with improved efficiency and stability," Nano. Energy, vol. 75, p. 104892, 2020.

[85] J. L. Knutson, J. D. Martin, and D. B. Mitzi, "Tuning the band gap in hybrid tin iodide perovskite semiconductors using structural templating," Inorg. Chem., vol. 44, pp. 4699-4705, 2005.

[86] C. Katan, L. Pedesseau, M. Kepenekian, A. Rolland, and J. Even, "Interplay of spin-orbit coupling and lattice distortion in metal substituted 3D tri-chloride hybrid perovskites," J. Mater. Chem. A, vol. 3, pp. 9232-9240, 2015.

[87] G. Liu, L. Kong, P. Guo, et al., "Two regimes of bandgap red shift and partial ambient retention in pressure-treated twodimensional perovskites," ACS Energy Lett., vol. 2, pp. 2518-2524, 2017.

[88] X. Li, J. Hoffman, W. Ke, et al., "Two-dimensional halide perovskites incorporating straight chain symmetric diammonium ions, $\left(\mathrm{NH}_{3} \mathrm{C}_{m} \mathrm{H}_{2 m} \mathrm{NH}_{3}\right)\left(\mathrm{CH}_{3} \mathrm{NH}_{3}\right)_{n-1} \mathrm{~Pb}_{n} \mathrm{I}_{3 n+1}(\mathrm{~m}=4-9 ; \mathrm{n}=1-4)$," J. Am. Chem. Soc., vol. 140, pp. 12226-12238, 2018.

[89] J.-C. Blancon, H. Tsai, W. Nie, et al., "Extremely efficient internal exciton dissociation through edge states in layered 2D perovskites," Science, vol. 355, pp. 1288-1292, 2017.

[90] A. Lemmerer and D. G. Billing, "Lead halide inorganic-organic hybrids incorporating diammonium cations," CrystEngComm, vol. 14, p. 1954, 2012.

[91] S. Dai, F. Zhao, Q. Zhang, et al., "Fused nonacyclic electron acceptors for efficient polymer solar cells," J. Am. Chem. Soc., vol. 139, pp. 1336-1343, 2017.

[92] J. Hu, I. W. H. Oswald, S. J. Stuard, et al., "Synthetic control over orientational degeneracy of spacer cations enhances solar cell efficiency in two-dimensional perovskites," Nat. Commun., vol. 10, p. 1276, 2019.

[93] F. Zhang, D. H. Kim, H. Lu, et al., "Enhanced charge transport in 2D perovskites via fluorination of organic cation," J. Am. Chem. Soc., vol. 141, pp. 5972-5979, 2019.

[94] T. Niu, J. Lu, M.-C. Tang, et al., "High performance ambient-airstable $\mathrm{FAPbl}_{3}$ perovskite solar cells with molecule-passivated Ruddlesden-Popper/3D heterostructured film," Energy Environ. Sci., vol. 11, pp. 3358-3366, 2018.

[95] D. B. Mitzi, C. A. Feild, W. T. A. Harrison, and A. M. Guloy, "Conducting tin halides with a layered organic-based perovskite structure," Nature, vol. 369, pp. 467-469, 1994.
[96] Z. Wang, Q. Lin, F. P. Chmiel, N. Sakai, L. M. Herz, and H. J. Snaith, "Efficient ambient-air-stable solar cells with 2D-3D heterostructured butylammonium-caesium-formamidinium lead halide perovskites," Nat. Energy, vol. 2, p. 17135, 2017.

[97] L. Protesescu, S. Yakunin, M. I. Bodnarchuk, et al., "Nanocrystals of cesium lead halide perovskites $\left(\mathrm{CsPbX}_{3}, \mathrm{X}=\mathrm{Cl}\right.$, $\mathrm{Br}$, and I): novel optoelectronic materials showing bright emission with wide color gamut," Nano Lett., vol. 15, pp. 3692-3696, 2015.

[98] P. Chen, Y. Bai, M. Lyu, J.-H. Yun, M. Hao, and L. Wang, "Progress and perspective in low-dimensional metal halide perovskites for optoelectronic applications," Solar RRL, vol. 2, p. 1700186 , 2018.

[99] W. Rehman, D. P. McMeekin, J. B. Patel, et al., "Photovoltaic mixed-cation lead mixed-halide perovskites: links between crystallinity, photo-stability and electronic properties," Energy Environ. Sci., vol. 10, pp. 361-369, 2017.

[100] M. Saliba, T. Matsui, K. Domanski, et al., "Incorporation of rubidium cations into perovskite solar cells improves photovoltaic performance," Science, vol. 354, pp. 206-209, 2016.

[101] N. Zhou, Y. Shen, L. Li, et al., "Exploration of crystallization kinetics in quasi two-dimensional perovskite and high performance solar cells," J. Am. Chem. Soc., vol. 140, pp. 459-465, 2017.

[102] M. Long, T. Zhang, D. Chen, et al., “Interlayer interaction enhancement in Ruddlesden-Popper perovskite solar cells toward high efficiency and phase stability," ACS Energy Lett., vol. 4, pp. 1025-1033, 2019.

[103] L. Gao, F. Zhang, X. Chen, et al., "Enhanced charge transport by incorporating formamidinium and cesium cations into twodimensional perovskite solar cells," Angew. Chem. Int. Ed., vol. 58, pp. 11737-11741, 2019.

[104] S. Tan, N. Zhou, Y. Chen, et al., "Effect of high dipole moment cation on layered 2D organic-inorganic halide perovskite solar cells," Adv. Energy Mater., vol. 9, p. 1803024, 2018.

[105] X. Lian, J. Chen, M. Qin, et al., "The second spacer cation assisted growth of a 2D perovskite film with oriented large grain for highly efficient and stable solar cells," Angew. Chem. Int. Ed., vol. 58, pp. 9409-9413, 2019.

[106] F. Hao, C. C. Stoumpos, P. Guo, et al., "Solvent-mediated crystallization of $\mathrm{CH}_{3} \mathrm{NH}_{3} \mathrm{Snl}_{3}$ films for heterojunction depleted perovskite solar cells," J. Am. Chem. Soc., vol. 137, pp. 11445-11452, 2015.

[107] Y. Wu, A. Islam, X. Yang, et al., "Retarding the crystallization of $\mathrm{Pbl}_{2}$ for highly reproducible planar-structured perovskite solar cells via sequential deposition," Energy Environ. Sci., vol. 7, pp. 2934-2938, 2014.

[108] P. Mao, J. Zhuang, Y. Wei, N. Chen, Y. Luan, and J. Wang, “Origin and suppression of the graded phase distribution in Ruddlesden-Popper perovskite films for photovoltaic application," Solar RRL, vol. 3, p. 1800357, 2019.

[109] J. Qiu, Y. Zheng, Y. Xia, L. Chao, Y. Chen, and W. Huang, "Rapid crystallization for efficient 2D Ruddlesden-Popper (2DRP) perovskite solar cells," Adv. Funct. Mater., vol. 29, p. 1806831, 2019.

[110] C. C. Stoumpos, C. D. Malliakas, and M. G. Kanatzidis, "Semiconducting tin and lead iodide perovskites with organic cations: phase transitions, high mobilities, and near-Infrared photoluminescent properties," Inorg. Chem., vol. 52, pp. 9019-9038, 2013. 
[111] Z. Chen, M. Liu, Z. Li, et al., "Stable Sn/Pb-based perovskite solar cells with a coherent 2D/3D interface," iScience, vol. 9, pp. 337-346, 2018.

[112] J. Lu, L. Jiang, W. Li, et al., “Diammonium and monoammonium mixed-organic-cation perovskites for high performance solar cells with improved stability," Adv. Energy Mater., vol. 7, p. 1700444, 2017.

[113] F. Hao, C. C. Stoumpos, R. P. H. Chang, and M. G. Kanatzidis, "Anomalous band gap behavior in mixed $\mathrm{Sn}$ and $\mathrm{Pb}$ perovskites enables broadening of absorption spectrum in solar cells," J. Am. Chem. Soc., vol. 136, pp. 8094-8099, 2014.

[114] W. Ke, I. Spanopoulos, Q. Tu, et al., "Ethylenediammoniumbased "hollow" $\mathrm{Pb} / \mathrm{Sn}$ perovskites with ideal band gap yield solar cells with higher efficiency and stability," J. Am. Chem. Soc., vol. 141, pp. 8627-8637, 2019.

[115] Z. Chen, X. Zheng, F. Yao, J. Ma, C. Tao, and G. Fang, "Methylammonium, formamidinium and ethylenediamine mixed triple-cation perovskite solar cells with high efficiency and remarkable stability," J. Mater. Chem. A, vol. 6, pp. 17625-17632, 2018.

[116] C. Ma, D. Shen, B. Huang, et al., "High performance lowdimensional perovskite solar cells based on a one dimensional lead iodide perovskite," J. Mater. Chem. A, vol. 7, pp. 8811-8817, 2019.

[117] T. Niu, J. Lu, X. Jia, et al., "Interfacial engineering at the 2D/3D heterojunction for high-performance perovskite solar cells," Nano Lett., vol. 19, pp. 7181-7190, 2019.

[118] Y. Lin, Y. Bai, Y. Fang, et al., "Enhanced thermal stability in perovskite solar cells by assembling 2D/3D stacking structures,” J. Phys. Chem. Lett., vol. 9, pp. 654-658, 2018.
[119] Q. Yao, Q. Xue, Z. Li, et al., “Graded 2D/3D perovskite heterostructure for efficient and operationally stable MA-Free perovskite solar cells," Adv. Mater., vol. 32, p. 2000571, 2020.

[120] T. Zhao, C.-C. Chueh, Q. Chen, A. Rajagopal, and A. K. Y. Jen, "Defect passivation of organic-inorganic hybrid perovskites by diammonium iodide toward high-performance photovoltaic devices," ACS Energy Lett., vol. 1, pp. 757-763, 2016.

[121] T. Niu, W. Zhu, Y. Zhang, et al., "D-A-ח-A-D-type dopant-free hole transport material for low-cost, efficient, and stable perovskite solar cells," Joule, vol. 5, pp. 249-269, 2021.

[122] D. Lin, T. Zhang, J. Wang, et al., "Stable and scalable 3D-2D planar heterojunction perovskite solar cells via vapor deposition," Nanomater. Energy, vol. 59, pp. 619-625, 2019.

[123] Y. Hu, Y. Chu, Q. Wang, et al., "Standardizing perovskite solar modules beyond cells," Joule, vol. 3, pp. 2076-2085, 2019.

[124] M. Zhou, C. Fei, J. S. Sarmiento, and H. Wang, "Manipulating the phase distributions and carrier transfers in hybrid quasi-twodimensional perovskite films," Solar RRL, vol. 3, p. 1800359, 2019.

[125] Y. Wei, H. Chu, Y. Tian, et al., "Reverse-graded 2D RuddlesdenPopper perovskites for efficient air-stable solar cells," Adv. Energy Mater., vol. 9, p. 1900612, 2019.

[126] Y.-W. Jang, S. Lee, K. M. Yeom, et al., "Intact 2D/3D halide junction perovskite solar cells via solid-phase in-plane growth," Nat. Energy, vol. 6, pp. 63-71, 2021.

[127] X. Zheng, Y. Hou, C. Bao, et al., "Managing grains and interfaces via ligand anchoring enables $22.3 \%$-efficiency inverted perovskite solar cells," Nat. Energy, vol. 5, pp. 131-140, 2020.

[128] T. Duong, H. Pham, T. C. Kho, et al., "High efficiency perovskitesilicon tandem solar cells: effect of surface coating versus bulk incorporation of 2D perovskite," Adv. Energy Mater., vol. 10, p. 1903553, 2020. 